\title{
Solar wind control of plasma number density in the near-Earth plasma sheet: three-dimensional structure
}

\author{
D. Nagata ${ }^{1}$, S. Machida ${ }^{1}$, S. Ohtani ${ }^{2}$, Y. Saito ${ }^{3}$, and T. Mukai ${ }^{3}$ \\ ${ }^{1}$ Department of Geophysics, Kyoto University, Kyoto 606-8502, Japan \\ ${ }^{2}$ Applied Physics Laboratory, Johns Hopkins University, Laurel, MD 20723, USA \\ ${ }^{3}$ Institute of Space and Astronautical Science, Japan Aerospace Exploration Agency, Sagamihara, Kanagawa 229-8510, Japan
}

Received: 30 March 2008 - Revised: 8 October 2008 - Accepted: 17 November 2008 - Published: 11 December 2008

\begin{abstract}
The plasma number density in the near-Earth plasma sheet depends on the solar wind number density and the north-south component of interplanetary magnetic field (IMF $B_{z}$ ) with time lag and duration of several hours. We examined the three-dimensional structure of such dependences by fitting observations of plasma sheet and solar wind to an empirical model equation. Analyses were conducted separately for northward and southward IMF conditions. Effects of solar wind speed and IMF orientation were also examined by further subdivision of the dataset. Based on obtained results, we discuss (i) the relative contribution of the ionosphere and solar wind to plasma sheet mass supply, (ii) the entry mechanisms for magnetosheath particles, and (iii) the plasma transport in the plasma sheet. We found that solar wind number density dependence is weaker and IMF $B_{z}$ dependence is stronger for faster solar wind with southward IMF, which suggests the contribution of ionospheric particles. Further from the Earth, different interplanetary conditions result in different structures of solar wind dependence, which indicate different solar wind entry mechanisms: (1) southward IMF results in a strong dependence on solar wind number density in the flank high-latitude region, (2) slow solar wind with northward IMF leads to lower-latitude peaks of solar wind number density dependence in the flank region, (3) fast solar wind with northward IMF results in a strong dependence on solar wind number density at the down-tail dusk flank equator, and (4) solar wind number density dependence is stronger in the downstream of quasi-parallel bow shock. These features are attributable to (1) low-latitude dayside reconnection entry, (2) high-latitude dayside reconnection entry, (3) entry due to decay of Kelvin-Helmholtz vortices, and (4) diffusive entry mediated by kinetic Alfven waves, respectively. Effect of IMF $B_{z}$ and its time lags show plasma sheet reconfiguration associated with enhanced convective trans-
\end{abstract}

Correspondence to: D. Nagata

(nagata@kugi.kyoto-u.ac.jp) port under southward IMF. Duration of IMF $B_{z}$ effect under northward IMF is interpreted in terms of turbulent diffusive transport.

Keywords. Magnetospheric physics (Magnetotail boundary layers; Plasma sheet; Solar wind-magnetosphere interactions)

\section{Introduction}

The sources of particles for the terrestrial plasma sheet are the fast flowing interplanetary medium, solar wind, and the terrestrial ionosphere. Both sources are known to be able to supply sufficient amount of particles to the plasma sheet (e.g. Chappell, et al., 1987; Walker et al., 2003; Moore et al., 2005). The study of mass transport in the plasma sheet from these two sources to the inner magnetosphere is a major topic in solar-terrestrial physics.

As for the ionospheric source, ion outflow from the highlatitude ionosphere strongly depends on flux of solar UV and solar wind parameters (e.g. Cully et al., 2003b). Subsequent supply of thermal ions from the high-latitude ionosphere to the central plasma sheet was estimated by particle tracing in a model electromagnetic field and in situ observations at low altitudes (e.g. Cully et al., 2003a). On the other hand, it is difficult to directly observe and evaluate ion supply from solar wind to the plasma sheet. Instead, correlation between solar wind parameters and plasma sheet number density has been examined for the various solar wind conditions to examine the solar wind particle supply.

Two solar wind parameters are known to correlate with the plasma sheet number density. One is the solar wind number density and the other is the north-south component of interplanetary magnetic field (IMF $B_{z}$ ). The plasma sheet number density positively correlates with the solar wind number density in a power law form (Borovsky et al., 1998). IMF

Published by Copernicus Publications on behalf of the European Geosciences Union. 

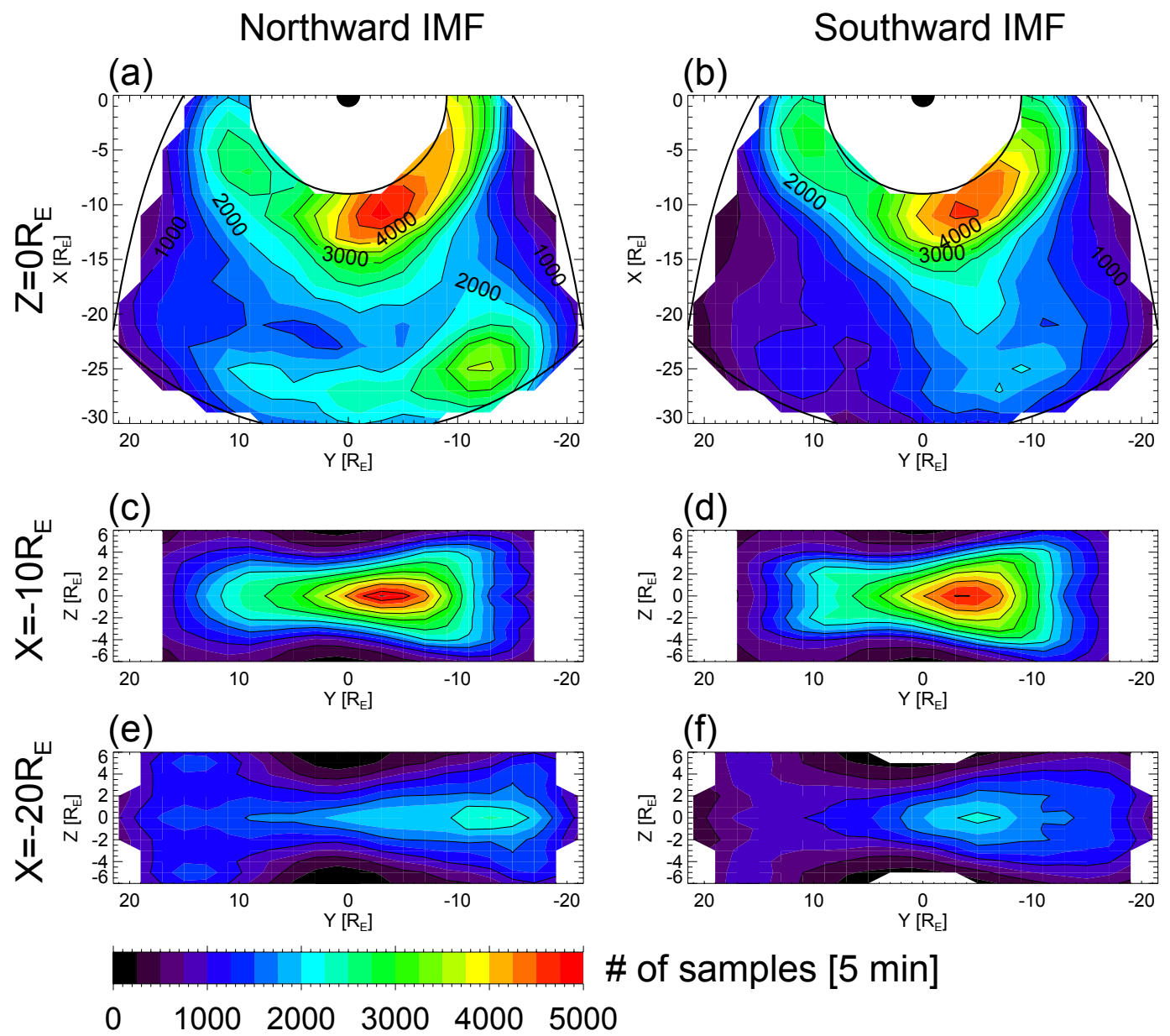

\section{\# of samples [5 min]}

Fig. 1. Distributions of data amount assigned for each grid for the northward and southward IMF conditions. The first row is $X Y$ distribution in the equatorial plane $\left(Z=0 R_{E}\right)$ and lower rows are $Y Z$ distributions in the $X=-10$ and $-20 R_{E}$ planes.

$B_{z}$ is important to understand the variation of plasma sheet number density under the same level of solar wind number density condition: northward IMF condition leads to cool and dense plasma sheet (Terasawa et al., 1997). There are several-hour time lags between variations of solar wind and plasma sheet and they are crucial to understand the transport path of magnetosheath particles into and through the plasma sheet (Terasawa et al., 1997; Borovsky et al., 1998).

As time advances, accumulation of data enabled researchers to investigate the two-dimensional structures of the equatorial plasma sheet properties in several ways. Effects of IMF $B_{z}$ including its duration have been investigated by averaging remote and in situ observations (Wing and Newell, 2002; Wing et al., 2005; Wang et al., 2006). Tsyganenko and Mukai (2003) developed a global analytic function for plasma sheet number density, which depends on the solar wind number density, speed, and IMF $B_{z}$ averaged over $30 \mathrm{~min}$ before plasma sheet observations. We also examined the effects of solar wind number density, IMF $B_{z}$, and its time lag simultaneously by fitting solar wind and plasma sheet observations to a local analytic function (Nagata et al., 2007).

If velocity distribution of particles is isotropic and neither source nor loss of particles exists, plasma number density is constant along magnetic field line. In that case, we can estimate the three-dimensional distribution of plasma number density from the equatorial distribution or from low-altitude particle observations with an accurate magnetic field model. However, there are various particle sources such as auroral outflow and magnetosheath particle entry across the magnetopause. Moreover modeling of global magnetic field is still under way of sophistication (Tsyganenko and Sitnov, 2007).

Kaufmann et al. (2002) constructed a three-dimensional plasma sheet model based on long-term in situ observations under the assumption of steady-state force balance. They revealed numerous aspects of plasma sheet such as temperature anisotropy, electric currents, magnetization of particles, flux tube particle content, and transport of magnetic flux and 

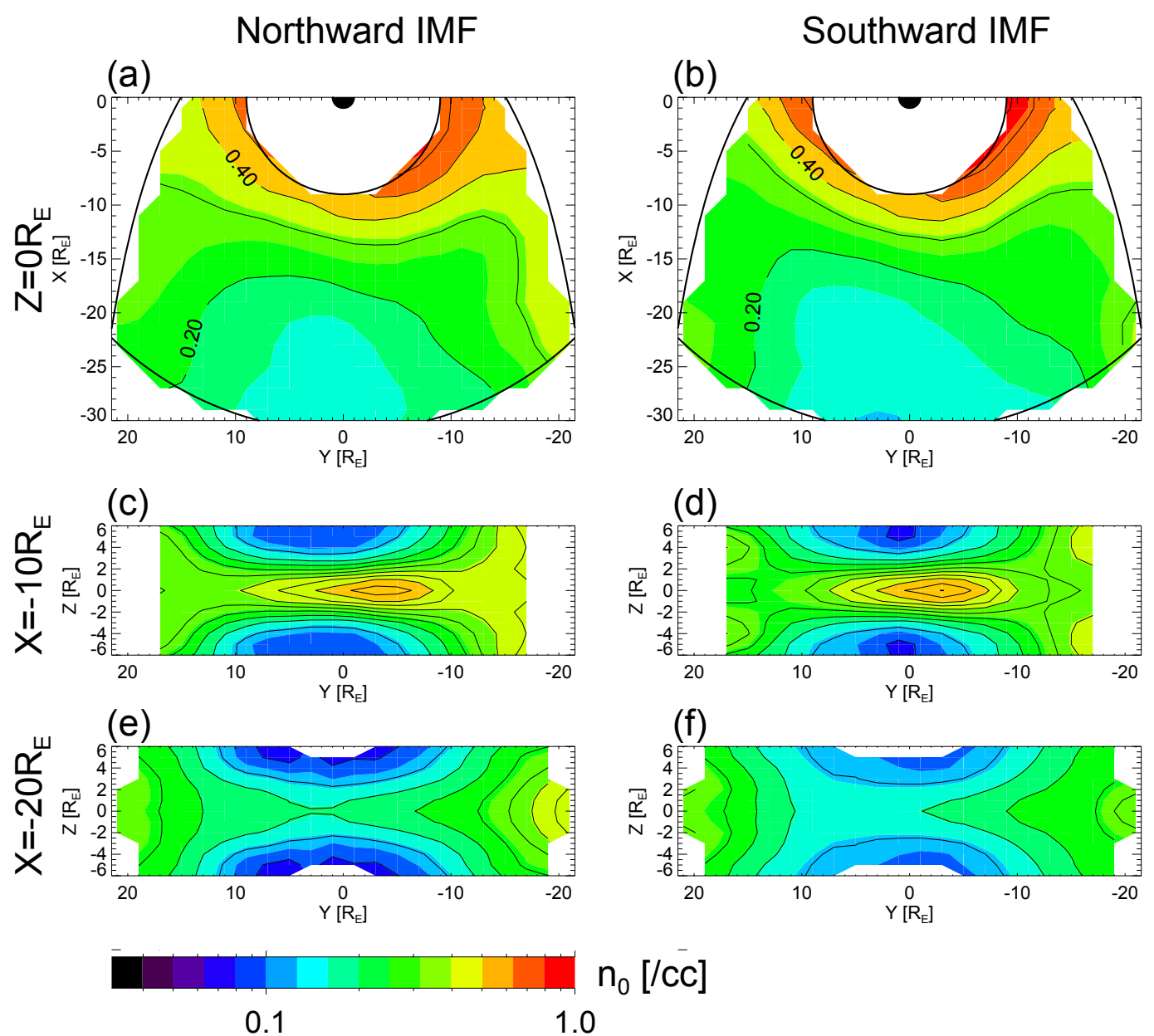

Fig. 2. Distribution of plasma number density $n_{0}$ for the northward and southward IMF conditions. Format is the same as Fig. 1 .

particle (Kaufmann et al., 2003, 2004, 2005, 2006). A series of their works showed that three-dimensional analysis sheds new light upon plasma sheet research.

In this paper, we examine three-dimensional structures of dependences of plasma sheet number density on solar wind number density and IMF $B_{z}$ by fitting the solar wind and plasma sheet observations to an empirical model equation. Time lag and duration of IMF $B_{z}$ effect are concurrently explored by optimizing the correlation coefficient of the fit. In order to resolve structures in the direction normal to the neutral sheet, we utilized an empirical model of neutral sheet position developed by Tsyganenko and Fairfield (2004). Analyses were conducted separately for northward and southward IMF conditions. Dependences on solar wind speed and IMF orientation are investigated by subdivision of the dataset. Based on obtained results, we discuss (1) the contribution of ionospheric particles, (2) the entry mechanisms for magnetosheath particles into the plasma sheet, and (3) the plasma transport in the near-Earth plasma sheet.

This paper is structured as follows. In the following section, dataset and method utilized in this study are explained.
Results of analyses are presented in Sect. 3. In Sect. 4, we discuss the limitations of analyses and the implications of results. Conclusions are summarized in Sect. 5.

\section{Analysis}

\subsection{Dataset}

Observations of solar wind and plasma sheet from January 1995 to November 2005 are utilized in this study. Solar wind data comes from five-minute resolution OMNI data. All solar wind data is time-shifted to the Earth's bow shock nose. GEOTAIL provides plasma and magnetic field data in the near-Earth plasma sheet. Plasma parameters are obtained by the LEP-EAI (Mukai et al., 1994) and the EPIC-STICS (Williams et al., 1994) instruments. The energy range from $20 \mathrm{eV} / \mathrm{q}$ to $270 \mathrm{keV} / \mathrm{q}$ is covered by these instruments. All particles detected by the LEP-EAI are assumed to be protons. Magnetic field data is measured by the MGF experiment (Kokubun et al., 1994). 
$Z=0 \operatorname{Re}$
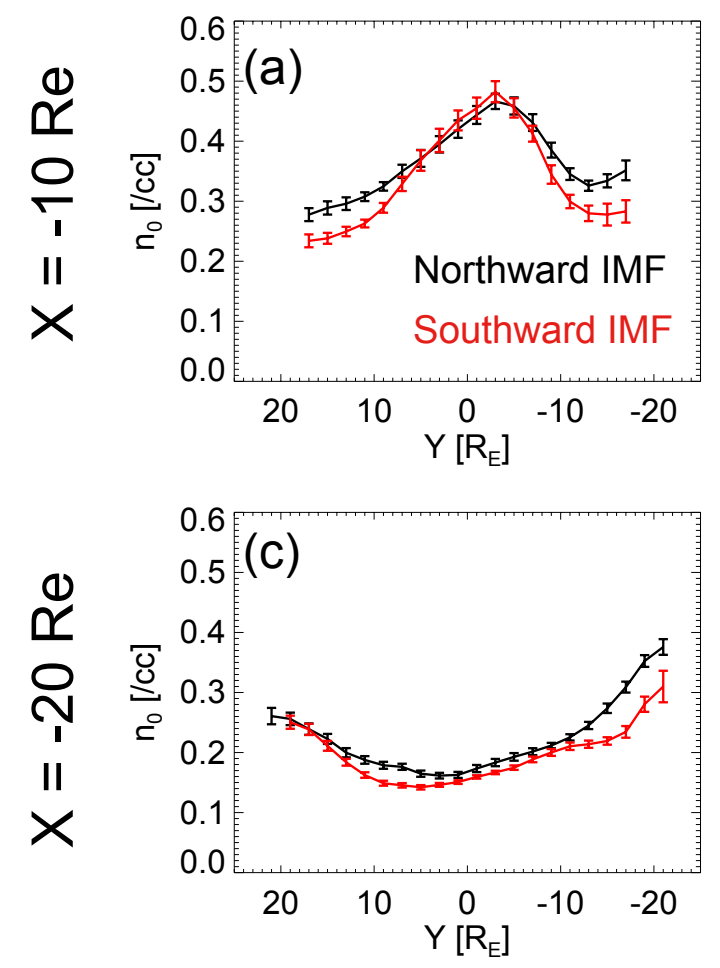

$Z=4 \operatorname{Re}$
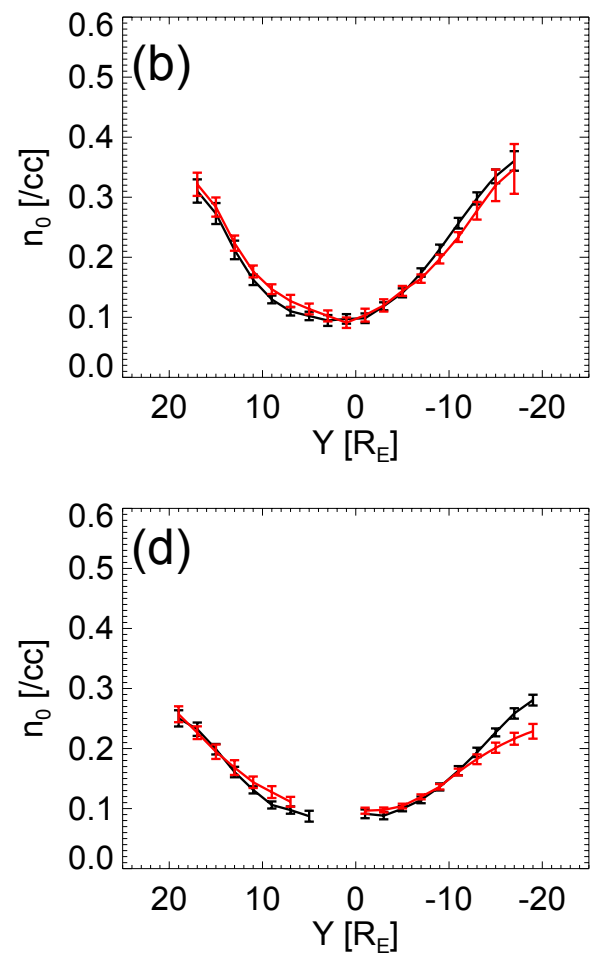

Fig. 3. $Y$ profiles of plasma number density $n_{0}$ at $X=-10$ and $-20 R_{E}$ for the northward and southward IMF conditions. Error bar is twice of standard error.

We use the Geocentric Solar Wind (GSW) coordinate system to represent the GEOTAIL observations. The GSW coordinate system is analogous to the Geocentric Solar Magnetospheric (GSM) coordinate system except that the direction of $\mathrm{X}$-axis is anti-parallel to the direction of solar wind flow. For the Z-coordinate of spacecraft position, we use deviation from the model neutral sheet of Tsyganenko and Fairfield (2004). To improve statistics, all data are folded about the model neutral sheet. Our investigation is limited to the nightside $\left(X<0 R_{E}\right)$ and the radial distance of 9 to $30 R_{E}$ from the Earth.

We use the following criteria to select plasma sheet data from GEOTAIL observations. (1) Ion temperature $T_{\text {ion }}>500 \mathrm{eV}$ to exclude magnetosheath and lobe observations, (2) ion flow speed $|V|<100 \mathrm{~km} / \mathrm{s}$ to remove magnetosheath and fast flows such as bursty bulk flows and plasmoids. Data obtained outside of the model magnetopause (Shue et al., 1998) with solar wind ram pressure $P d=2 \mathrm{nPa}$ and IMF $B_{z}=0 \mathrm{nT}$ are also discarded. Plasma sheet data are averaged over 5-min intervals.

The dataset is divided into two subsets based on the sign of IMF $B_{z}$ averaged over a 10-h interval prior to the plasma sheet observation. If the averaged IMF $B_{z}$ is positive, the data is categorized as northward IMF condition. Data with negative averaged IMF $B_{z}$ is categorized as southward IMF condition. Since autocorrelation time of solar wind number density is about $11 \mathrm{~h}$ (Borovsky et al., 1998), solar wind density can be assumed to be quasi-steady within this time scale except for abrupt changes associated with discontinuities in the solar wind.

In order to examine the effects of solar wind speed and IMF orientation, we also subdivide the dataset. Subdivisions are made for both northward and southward IMF datasets separately. Data with solar wind speed larger than $420 \mathrm{~km} / \mathrm{s}$ is regarded as the fast solar wind data, and the rest of data is categorized as slow solar wind data. The value of $420 \mathrm{~km} / \mathrm{s}$ is the median value of solar wind speed for our dataset. IMF orientation is evaluated by azimuth angle of IMF $\phi_{\text {imf }}=\tan ^{-1}\left(b_{y} / b_{x}\right)$, where $b_{x}$ and $b_{y}$ are X- and Ycomponent of IMF, respectively. If $\phi_{\mathrm{imf}}$ is positive, the data is categorized as ortho-Parker orientation, whereas negative $\phi_{\text {imf }}$ data is categorized as Parker orientation data. About $70 \%$ of the samples are Parker orientation data.

Plasma sheet data is spatially divided based on (X, Y, Z) coordinates. We set $2 R_{E} \times 2 R_{E}$ grids in XY plane. The grid width in $\mathrm{Z}$ direction is $1 R_{E}$. Samples within $5 R_{E}$ from a grid in XY plane and within $1 R_{E}$ in Z-direction are assigned to the grid. The distributions of assigned data amount for the northward and southward IMF subsets are shown in Fig. 1. One hundred samples are randomly chosen from the assigned 


\section{Northward IMF}

(a)

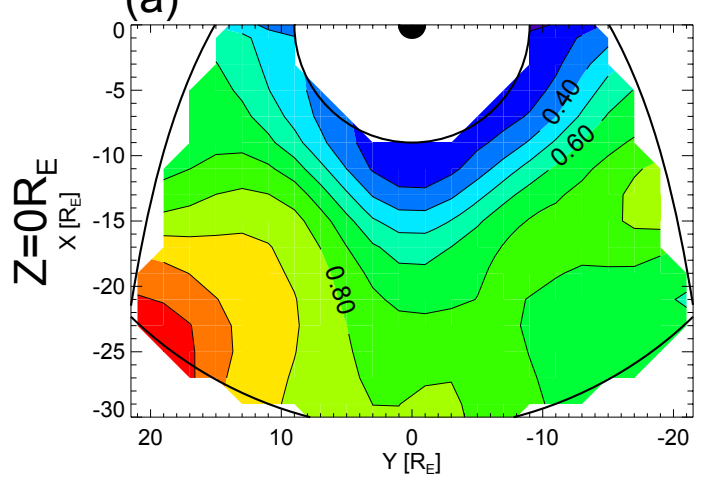

(c)

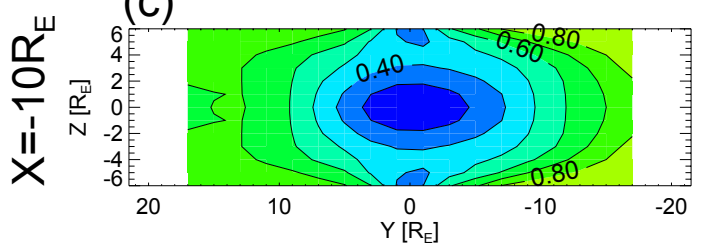

(e)
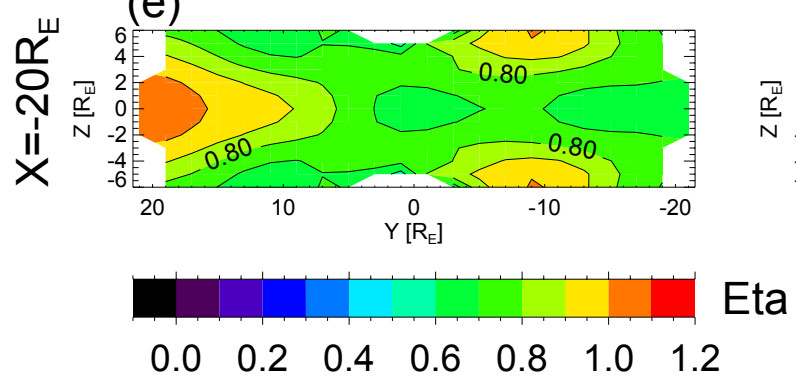

\section{Eta}

Southward IMF

(b)

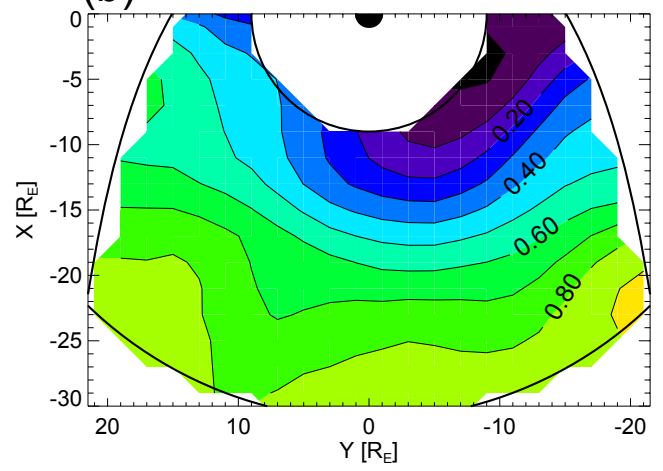

(d)

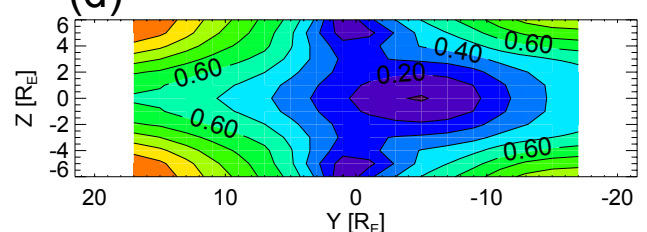

(f)

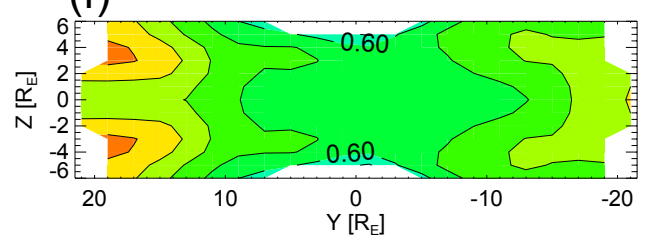

Fig. 4. Distributions of solar wind number density dependence parameter $\eta$ for the northward and southward IMF conditions. Format is the same as Fig. 1.

data for fitting, which is described in the following subsection, in order to reduce the effect of uneven data distribution.

\subsection{Method}

Plasma sheet and solar wind observations are fitted to a model equation to evaluate parameters which represent the dependences of plasma sheet number density on solar wind parameters. The model equation was determined on the assumption that the plasma sheet number density depends on the solar wind number density in a power law form (Borovsky et al., 1998) and on IMF $B_{z}$ in a exponential form (Terasawa et al., 1997). We did not include the factor of solar wind speed because the solar wind number density and velocity have negative correlation and are not suitable for independent variables of regression analysis. Thus we set the model equation as follows.

$n_{p s}=n_{0}\left(n_{s w}^{\left(t_{1}, t_{2}\right)} / n_{s w 0}\right)^{\eta} \exp \left(\alpha \cdot b_{z}^{\left(t_{1}, t_{2}\right)}\right)$ where, $n_{p s}$ is the plasma sheet number density. The value of $n_{s w 0}$ is set to be a typical value of $5 / \mathrm{cc}$. The parameters $n_{s w}^{\left(t_{1}, t_{2}\right)}$ and $b_{z}^{\left(t_{1}, t_{2}\right)}$ are the solar wind number density and IMF $B_{z}$ averaged from $t_{1} \mathrm{~h}$ to $t_{2} \mathrm{~h}$ prior to each plasma sheet observation $\left(t_{1}=1,2, \ldots, 10\right.$ and $\left.t_{2}=0,1, \ldots, t_{1}-1\right)$ :

$$
\begin{aligned}
& n_{s w}^{\left(t_{1}, t_{2}\right)}(t)=\frac{1}{t_{1}-t_{2}} \int_{t-t_{1}}^{t-t_{2}} n_{s w}\left(t^{\prime}\right) d t^{\prime} \\
& b_{z}^{\left(t_{1}, t_{2}\right)}(t)=\frac{1}{t_{1}-t_{2}} \int_{t-t_{1}}^{t-t_{2}} b_{z}\left(t^{\prime}\right) d t^{\prime}
\end{aligned}
$$

The parameter $n_{0}$ represents the plasma number density under the condition of $n_{s w}=5 / \mathrm{cc}$ and IMF $B_{z}=0 \mathrm{nT}$. Dependences of plasma sheet number density on solar wind number density and IMF $B_{z}$ are represented by the parameters $\eta$ and $\alpha$, respectively. Taking logarithm of Eq. (1), we used the following equation for the least square fit.

$$
\log n_{p s}=\log n_{0}+\eta \log \left(n_{s w}^{\left(t_{1}, t_{2}\right)} / n_{s w 0}\right)+\alpha \cdot b_{z}^{\left(t_{1}, t_{2}\right)}
$$




\section{$Z=0 \mathrm{Re}$}
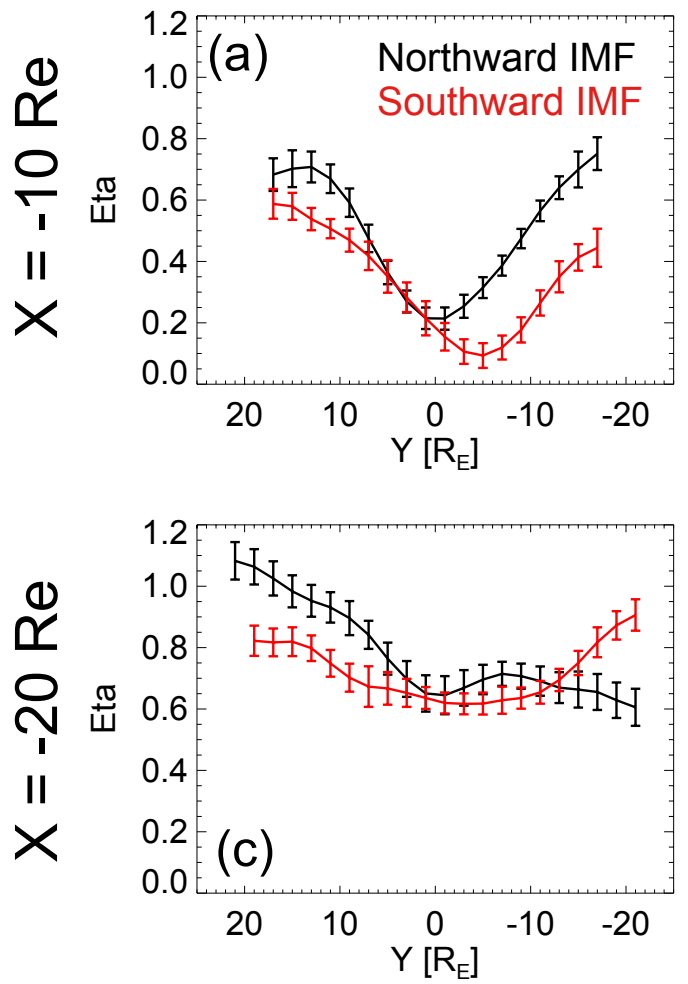

$Z=4 R e$
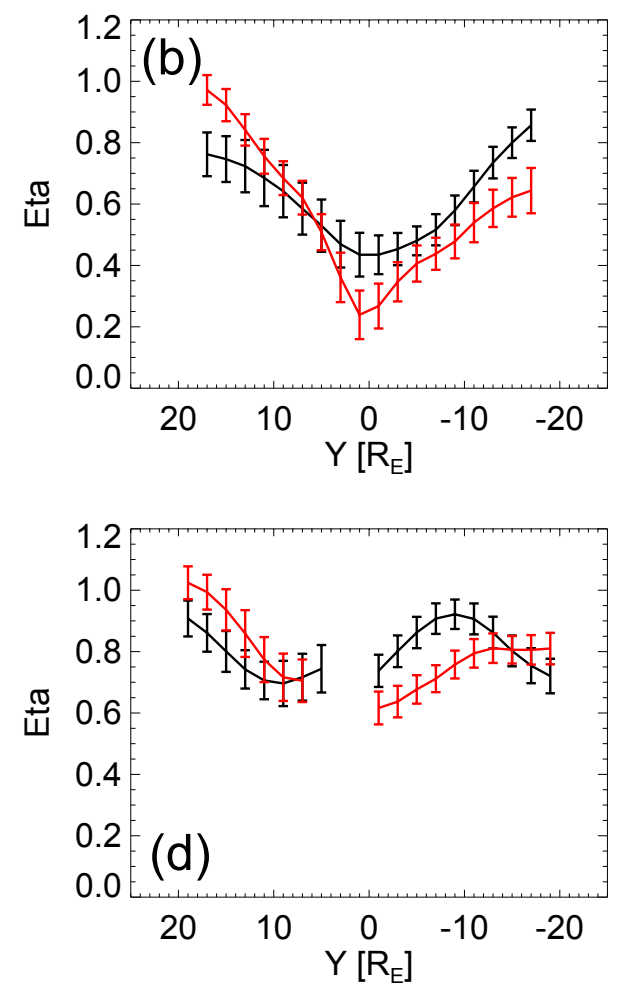

Fig. 5. $Y$ profiles of parameter $\eta$ for the northward and southward IMF conditions. Format is the same as Fig. 3. Error bar is Error bars $1-\sigma$ of least square fit.

To seek response time to the IMF $B_{z}$ effects, we repeated the fitting process described above for all pairs of $\left[t_{1}, t_{2}\right]$ for each subset, and selected the pair of $\left[t_{1}, t_{2}\right]$ which maximized the linear correlation coefficient between $\log \left(n_{p s} / n_{s w}^{\eta}\right)$ and $b_{z}$. This procedure was conducted for each grid. Coefficients of the fit shown in this paper are the results for selected pairs of $\left[t_{1}, t_{2}\right]$.

\section{Results}

In this section we present results of fitting described in the previous section. The main advantage of our method over previous ones is the possibility to investigate threedimensional structures. In addition to equatorial distributions of parameters, $Y Z$ cross-sections are also presented. We show here the results for the northward and southward IMF conditions. Results of further subdivided cases will be shown in Sect. 4.

\subsection{Basic structure of the near-Earth plasma sheet}

The parameter $n_{0}$ represents plasma number density expected under the condition of $n_{s w}=5 / \mathrm{cc}$ and null IMF $B_{z}$. The distributions under the northward and southward IMF condi- tions are similar, but have several differences (Fig. 2). In the equatorial plane $\left(Z=0 R_{E}\right)$, plasma number density increases closer to the Earth or the flank magnetopause. The normalized plasma number density is less than 0.8 /cc in the region analyzed. Maximum of plasma number density is located in the near-Earth region adjacent to the dawn terminator. The plasma number density minimizes at midnight in the distant region $\left(X=-30 R_{E}\right)$.

There are two differences between northward and southward IMF conditions. First, the value of $n_{0}$ is slightly $(<0.1 / \mathrm{cc})$ larger along the dawn flank magnetopause under northward IMF (Figs. 2a, 3a, c, and d). Second, plasma number density under southward IMF is lower in the premidnight region $\left(Y=5 R_{E}\right)$ than post-midnight region around $X=-20 R_{E}$ (Figs. $2 \mathrm{f}$ and $3 \mathrm{c}$ ), where magnetic reconnection is thought to occur.

From a three-dimensional viewpoint, the near-Earth plasma sheet seems to consist of two parts: "inner region" centered at midnight and "flank region" adjacent to the magnetopause (Fig. 2c-d). In the inner region, the plasma density structure aligns with magnetic field and plasma is denser in the lower latitude flux tube in XZ-plane (not shown). On the other hand, the flank region is thick in Z-direction along the flank magnetopause and has different structures. 

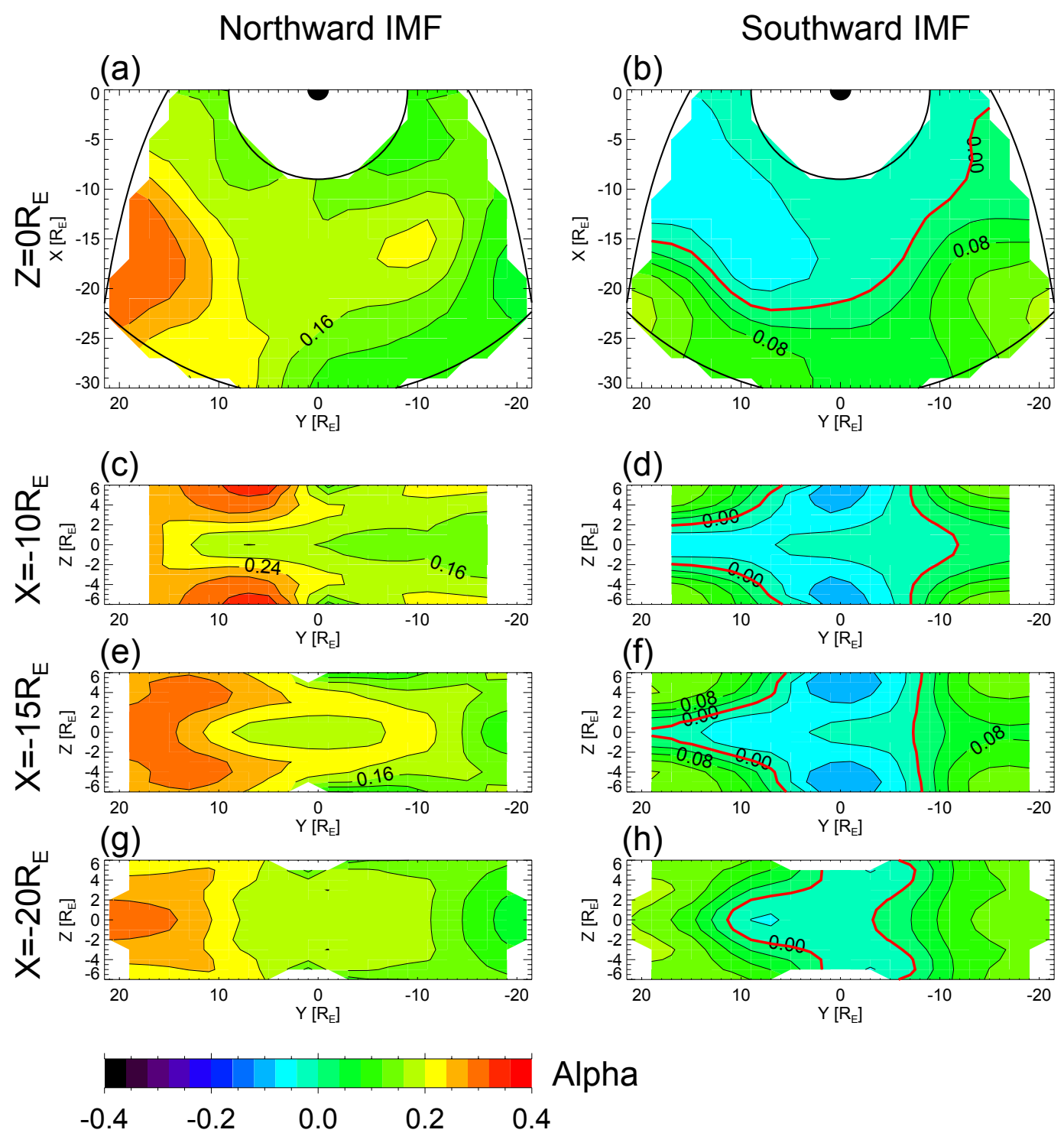

Fig. 6. Distributions of IMF $B_{Z}$ dependence parameter $\alpha$ for the northward (left) and southward (right) IMF conditions. The first row is $X Y$ distribution in the equatorial plane $\left(Z=0 R_{E}\right)$ and lower rows are $Y Z$ distributions in the $X=-10,-15$, and $-20 R_{E}$ planes.

In the near-Earth flank region $\left(|Y|>10 R_{E}\right.$ and $X>-15 R_{E}$ ), the plasma number density has its peak off equator under southward IMF condition (Fig. 2d), which is not obvious under northward IMF condition (Fig. 2c). This peak is emphasized by decrease of plasma number density at the equator rather than increase of plasma number density off equator (Fig. 3a-b). In the more distant part of the flank region $\left(X<-15 R_{E}\right)$, the peak in plasma number density is located in the neutral sheet under both IMF conditions. In this region, the dense plasma region connecting both flanks extends toward midnight (Fig. 2e).

\subsection{Dependence on solar wind number density}

The parameter $\eta$ represents the dependence of plasma sheet number density on solar wind number density. A large value of $\eta$ means a sensitive response of the plasma sheet number density to the solar wind number density variation. Physical interpretation of $\eta$ will be discussed in Sect. 4 in association with the mass source of plasma sheet. Here, we merely describe the distribution of $\eta$ and its differences between northward and southward IMF conditions.

As a global trend, the value of $\eta$ decreases from slightly larger than unity in the distant region to nearly zero in the near-Earth dawn region for both IMF conditions (Fig. 4ab). A closer look shows that there are several differences 
$Z=0 \operatorname{Re}$
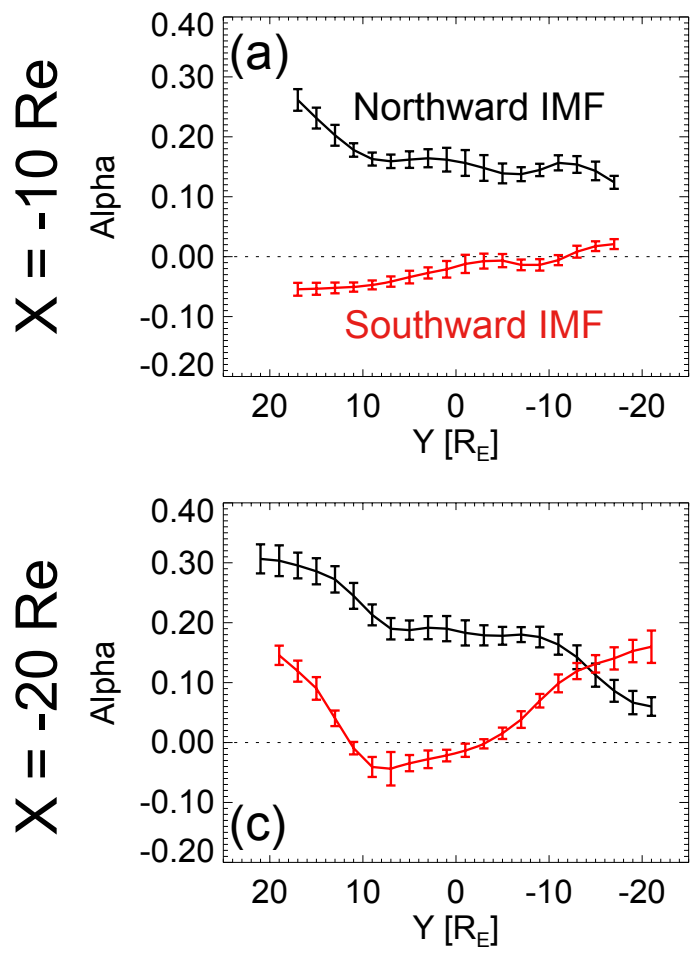

$Z=4 \operatorname{Re}$
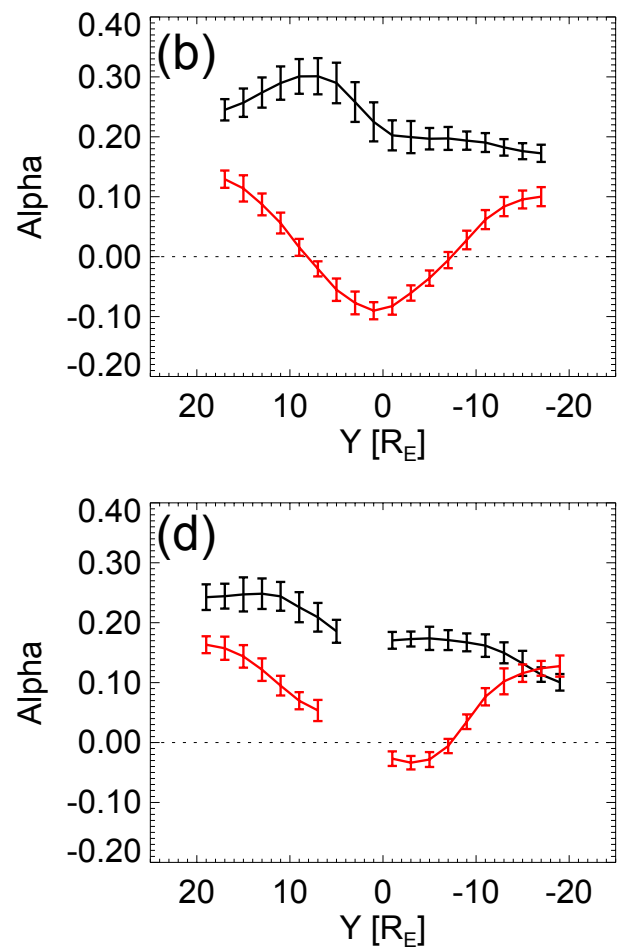

Fig. 7. $Y$ profiles of IMF $B_{z}$ dependence parameter $\alpha$ for the northward and southward IMF conditions. Format is the same as Fig. 3. Error bar is Error bars $1-\sigma$ of fit.

between distributions of $\eta$ in the equatorial plane for the northward and southward IMF conditions. First, the value of $\eta$ is larger in the near-Earth region $\left(X>-15 R_{E}\right)$ for the northward than southward IMF condition (Fig. 5a) and the $Y$ profile of $\eta$ is roughly symmetric for northward IMF whereas the dawn-dusk asymmetry of $\eta$ in the near-Earth region is strengthened under southward IMF condition. Second, in the more distant plasma sheet $\left(X<-20 R_{E}\right)$, the value of $\eta$ is larger in the dusk flank region than the dawn flank region for northward IMF whereas the distribution is more dawn-dusk symmetric for southward IMF (Fig. 5c).

As for the three-dimensional structure, we found several features which were not observable in the equatorial plane. Although the parameter $\eta$ is basically smaller at lower latitude, there are local minima of $\eta$ at the high latitude of the midnight region (Fig. 4c-d). These local minima are more prominent and have negatively larger values for southward IMF. Their positions are shifted a few Earth radii toward dusk. This fact reminds us that the auroral expansion most frequently initiates around 23:00 MLT (Frey et al., 2004). Thus, this feature is attributable to auroral outflow that supplies non-solar wind origin particles, which makes the solar wind number density dependence weak. This topic will be mentioned later in this section from a viewpoint of IMF $B_{z}$ dependence of plasma sheet number density.
In the dusk flank region, the value of $\eta$ has its peak off equator for southward IMF (Fig. 4e) and the peak is located at the equator for northward IMF (Fig. 4f). The value of $\eta$ is comparable for both IMF conditions although the position is different. In the dawn flank region, on the other hand, the value of $\eta$ does not maximize at the equator for both IMF conditions. These features will be interpreted from a perspective of magnetosheath particle entry in Sect. 4.

\subsection{Dependence on IMF $B_{z}$}

The parameter $\alpha$ represents the dependence on IMF $B_{z}$. A positive value of $\alpha$ means that plasma sheet is denser for the larger IMF $B_{z}$, whereas negative $\alpha$ means that the plasma sheet number density increases for the more negative value of IMF $B_{z}$. The distribution of $\alpha$ is totally different for each IMF condition (Figs. 6 and 7). The value of $\alpha$ is positive in the whole plasma sheet under northward IMF, whereas the value of $\alpha$ is negative in the near-Earth region under southward IMF. Thus, plasma number density increases everywhere in the plasma sheet for northward IMF whereas both increases and decreases of plasma number density occur under southward IMF.

In more detail, there are negative minima of $\alpha$ at the high latitude part of midnight under southward IMF (Fig. 6d, f). This means that the plasma number density in this region 

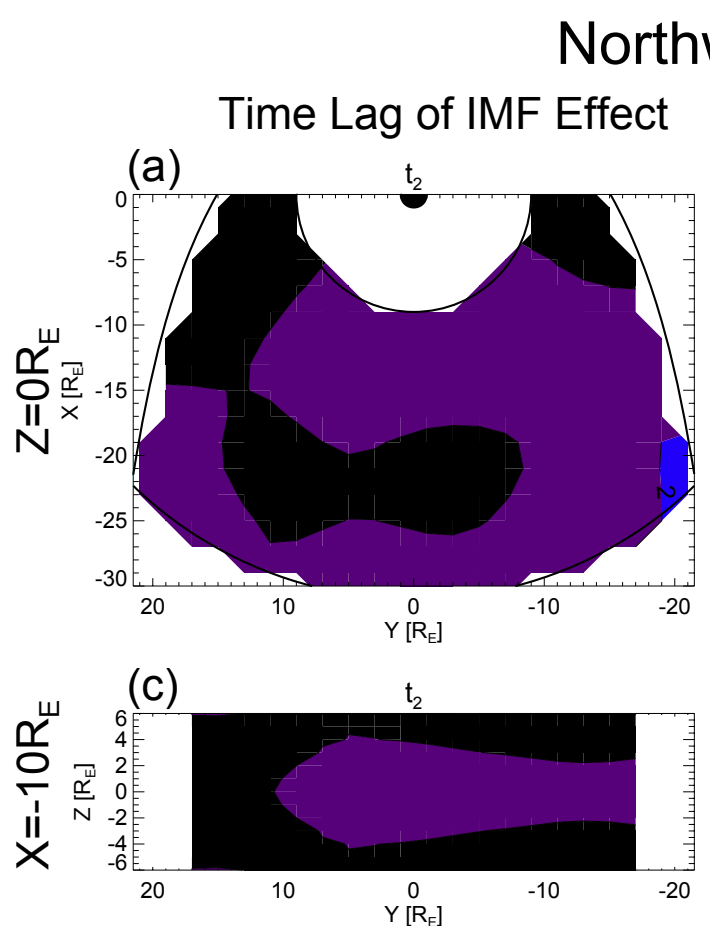

\section{ward IMF}
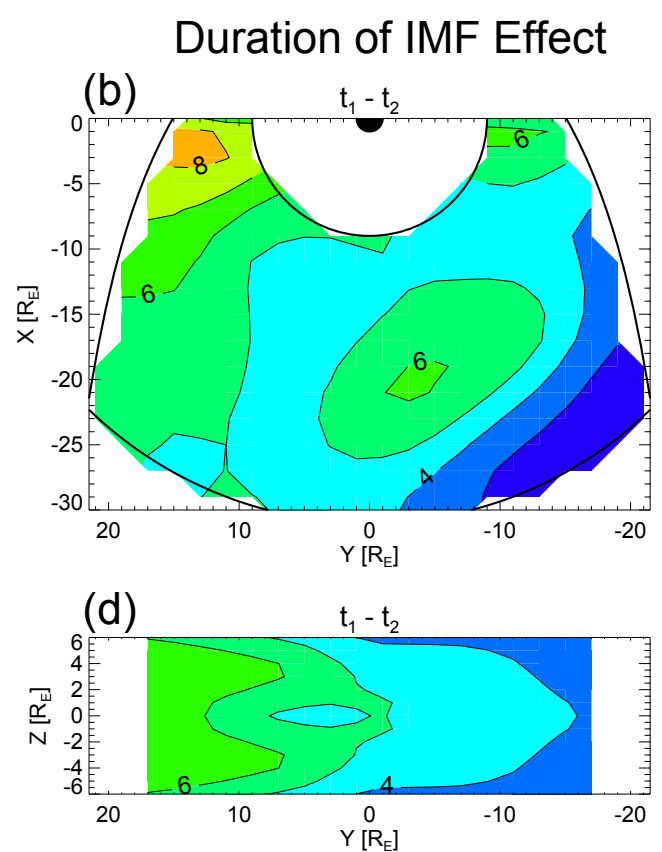

(e)
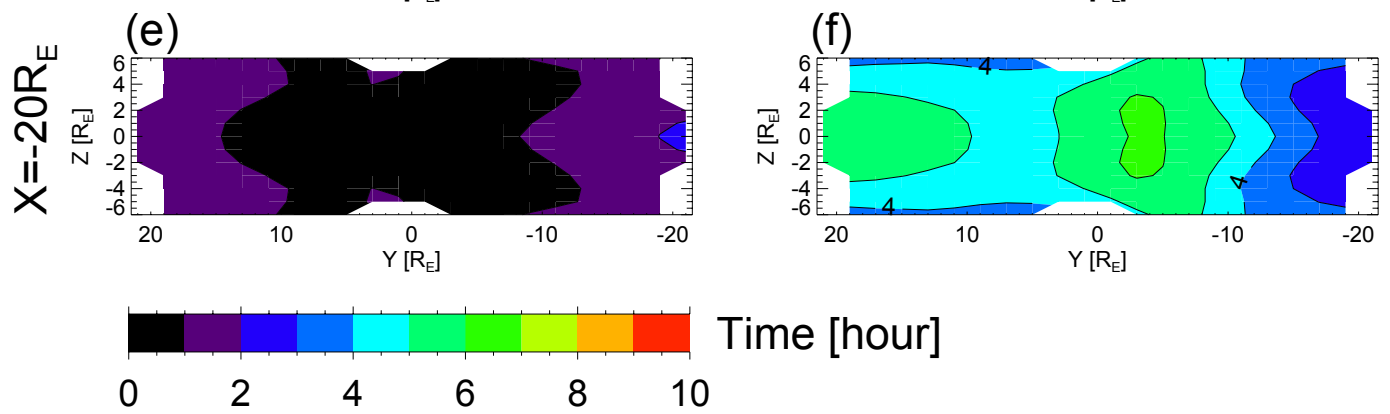

Time [hour]

Fig. 8. Distributions of time lag and duration of IMF $B_{z}$ effect for northward IMF condition. Format is the same as Fig. 1.

largely increases with the decreasing value of IMF $B_{z}$. This feature is consistent with the previous interpretation that auroral outflow results in smaller value of $\eta$ because the auroral outflow is more intense under southward IMF condition (Cully et al., 2003). The region of negative $\alpha$ is located around midnight and extends to the pre-midnight near-Earth region. In the flank region, the value of $\alpha$ is positively larger at the higher latitude region and magnitude is almost dawndusk symmetric for southward IMF (Fig. 7b).

Under northward IMF, the value of $\alpha$ is larger in the dusk region. The value of $\alpha$ is larger at the equator in the dusk region for $X<-15 R_{E}$ (Fig. $6 \mathrm{~g}$ ) whereas it is larger off equator in the region of $X>-15 R_{E}$ (Fig. 6c, e). The position of the peak also shifts toward the midnight meridian and it is roughly aligned with magnetic field line. A similar fieldaligned structure of cold dense plasma only in the dusk flank region is realized in the test particle simulation within global MHD field (T. E. Moore, private communication). On the other hand, the value of $\alpha$ is small and minimizes at the equa- tor along the dawn flank. This means that dawn side flank plasma sheet is less relevant thus more stable to variations of IMF $B_{z}$.

\subsection{Time lag and duration of IMF effect}

The parameters $t_{1}$ and $t_{2}$ represent the longest and shortest time length relative to plasma sheet observations between which the solar wind parameters are averaged to optimize the correlation between normalized plasma number density and IMF $B_{z}$. In other words, the IMF $B_{z}$ condition within $t_{2} \mathrm{~h}$ before the plasma sheet observations does not have close relation to the observed plasma sheet number density. Thus, the parameter $t_{2}$ is interpreted as the shortest time lag of IMF $B_{z}$ influences to appear. Similarly, the values of IMF $B_{z}$ prior to $t_{1} \mathrm{~h}$ before plasma sheet observation does not affect the observed plasma sheet number density and hence the parameter $t_{1}$ is taken as the longest time lag. Thus we assume that the effect of IMF $B_{z}$ takes $t_{2} \mathrm{~h}$ to occur and lasts for $t_{1}-t_{2} \mathrm{~h}$. 


\section{Southward IMF}
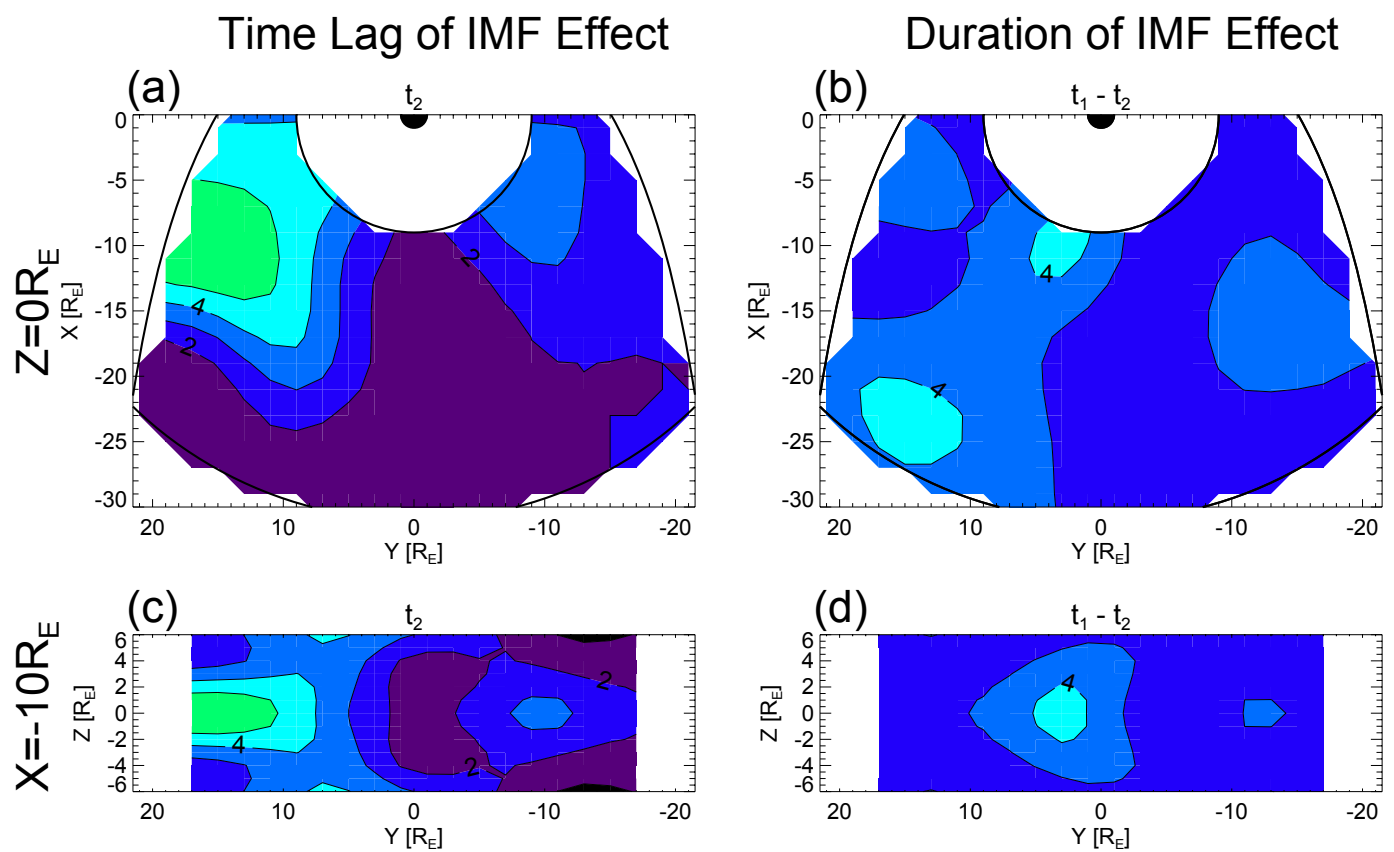

(e)
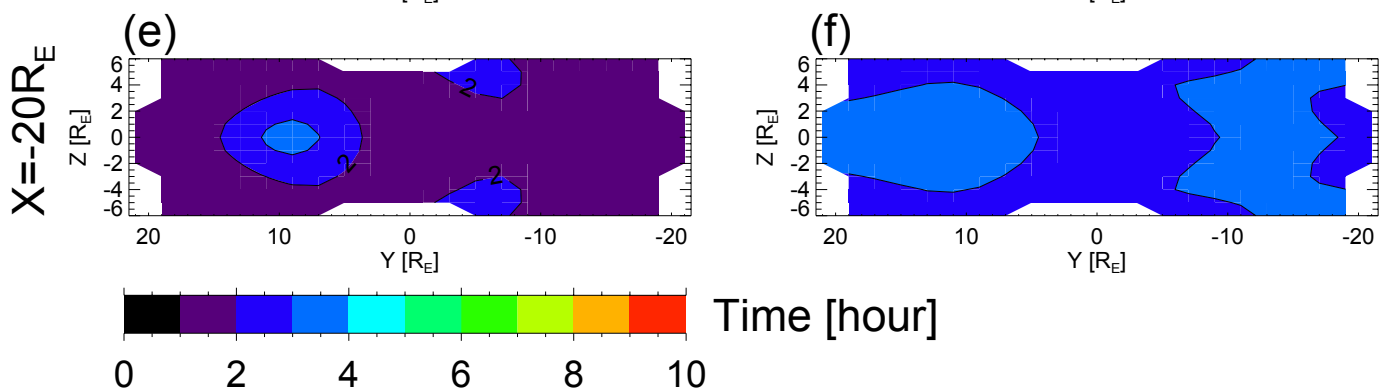

Fig. 9. Distributions of time lag and duration of IMF $B_{z}$ effect for southward IMF condition. Format is the same as Fig. 8 .

Distributions of time lag and duration of IMF $B_{z}$ effect are drastically different for the two IMF conditions (Figs. 8 and 9). Under northward IMF, the value of $t_{2}$ is short and ranges less than $2 \mathrm{~h}$. The value is smaller at higher latitude of flank in the near-Earth region and it also minimizes at midnight at $X<-20 R_{E}$. Because auto-correlation time of IMF $B_{z}$ is about $100 \mathrm{~min}$ (Borovsky et al., 1998), this difference is within error and not conclusive. The duration of IMF $B_{z}$ effect represented by $t_{1}-t_{2}$ ranges from 2 to $9 \mathrm{~h}$ and has its minimum downtail of the dawn flank $\left(X<-17 R_{E}\right)$ and its maximum at the near-Earth dusk flank $\left(X>5 R_{E}\right)$. There is also a local maximum around $(X, Y)=(-20,-3) R_{E}$.

Under southward IMF, the value of $t_{2}$ ranges up to $6 \mathrm{~h}$ and the value of $t_{1}-t_{2}$ is rather uniform and is about $3 \mathrm{~h}$. The value of $t_{2}$ is as small as $1 \mathrm{~h}$ at $r>25 R_{E}$ in the midnight near-Earth region. This time scale is comparable to the crosspolar-cap convection time in the ionosphere (Borovsky et al., 1998). The value of $t_{2}$ increases toward the near-Earth flanks under southward IMF. The distribution of $t_{2}$ is roughly mirror symmetric with respect to the noon-midnight meridian with larger value in the dusk side.

\subsection{Correlation coefficient of fit}

The distribution of absolute value of correlation coefficient of the fit is shown in Fig. 10. The value of correlation coefficient decreases toward the Earth and its distributions are almost similar to that of $\eta$. The value ranges from less than 0.8 and is as low as 0.1 in the near-Earth dawn region. This means that less than $60 \%$ of number density variation in the plasma sheet is explained by our modeling. Various sources of errors are discussed in Sect. 4.1

\section{Discussion}

In this section we discuss (1) the limitations of our analysis, (2) the contribution of ionospheric particles to the near-Earth plasma sheet, (3) the entry mechanisms of magnetosheath 

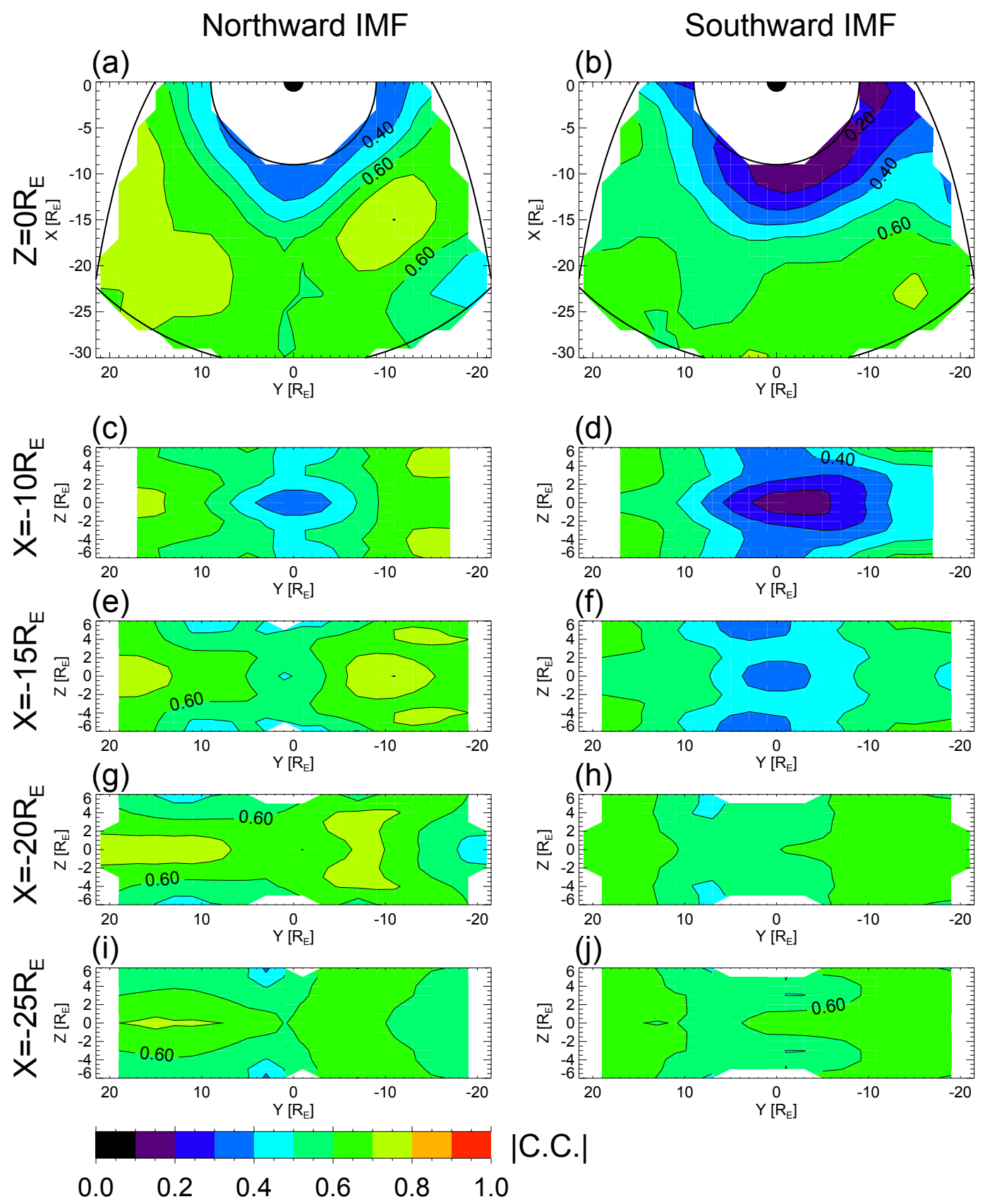

Fig. 10. Distribution of absolute value of correlation coefficient of the fit. Format is the same as Fig. 1.

particles into the near-Earth plasma sheet, and (4) the particle transport in the plasma sheet.

\subsection{Limitations of analysis}

Our analysis utilized a neutral sheet position model to evaluate the relative position of measurements from the neutral sheet. The model gives us statistically optimized estimate of the neutral sheet position. Finite difference between predicted and actual neutral sheet position is unavoidable for each observation. This deviation can lead to the error in the analysis. It is also known that the plasma sheet flaps (Sergeev et al., 2004, 2006) and oscillates in eigen mode (Vorwerk et al., 2004). These variations are not included in the neutral sheet position model and also lead to the error. Thinning and expansion of the plasma sheet associated with the magnetospheric substorm can also cause errors.

One of the difficulties in our analysis comes from the limited data amount to cover the vast area to be investigated. We set a large bin to increase the data amount assigned to each 


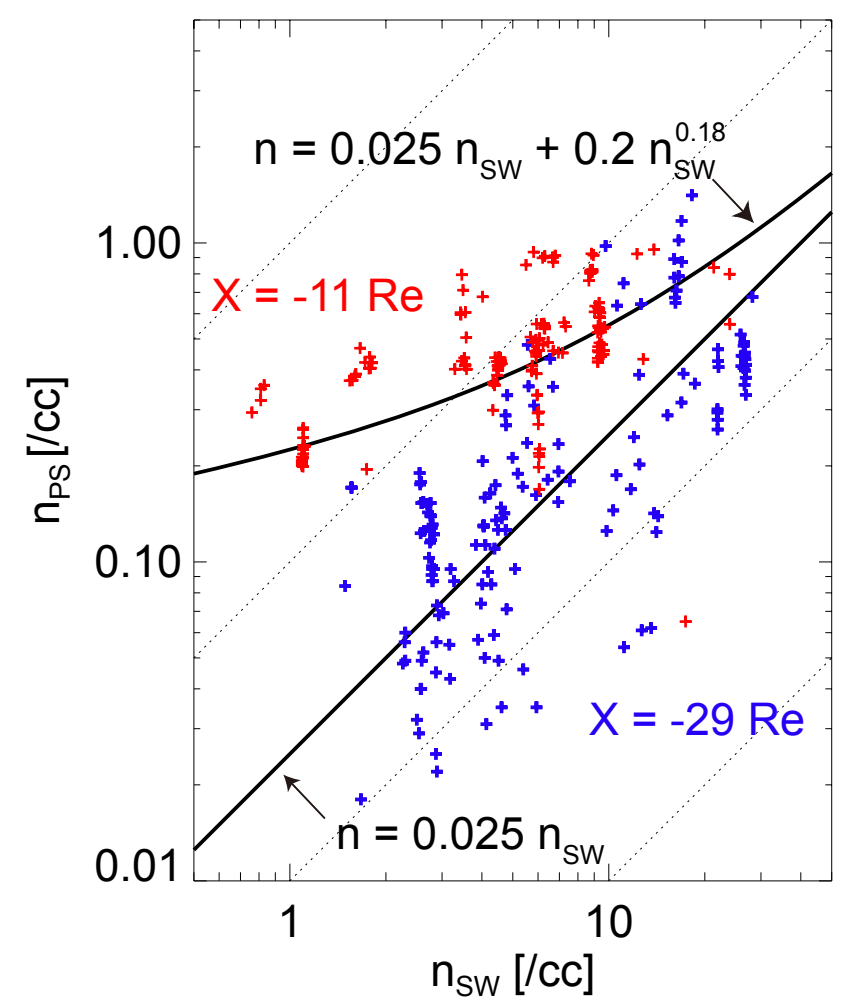

Fig. 11. Plasma sheet number density versus solar wind number density at the midnight meridian for different values of $X$. Red crosses indicate data point for $X=-11 R_{E}$ and blue crosses for $X=-29 R_{E}$. Line indicates the purely solar wind origin case and curve means mixture of solar wind origin and ionospheric origin plasma.

grid. This process results in smoothing of the spatial distributions. We also folded the observations with respect to the neutral sheet under the assumption of north-south symmetry in order to double the data amount. North-south asymmetry which can be caused for example by IMF $B_{y}$ or dipole tilt effects are not taken into account.

We divided the observations in two categories based on 10-h averaged value of IMF $B_{z}$. Since the autocorrelation time of IMF $B_{z}$ is $2.5 \mathrm{~h}$, each IMF subsets may contain several changes of IMF $B_{z}$ sign during $10 \mathrm{~h}$. Short timescale effects of IMF $B_{z}$ are not included in our analysis. Moreover, a whole sequence of magnetospheric substorm can be completed during the 10-h interval, thus phases of substorm are not distinguished. Further investigation and elaboration are needed to reveal such short timescale variations.

Plasma observations were obtained by the LEP-EAI and the EPIC-STICS instruments. Since the LEP-EAI is an electrostatic analyzer, mass and charge state of particles were not distinguished. If the number density of $\mathrm{O}^{+}$particles is calculated as they were protons, the value is one fourth of the true value. During intense storms and substorms, some part of plasma sheet can contain $\mathrm{O}^{+}$ions comparable to protons
(Lennartsson et al., 1992). The plasma number density is underestimated in such situations.

\subsection{Contribution of ionospheric particles}

Particles in the near-Earth plasma sheet are supplied from the ionosphere and the magnetosheath. These two sources have different dependences on the solar wind number density. Ion supply from the ionosphere weakly depends on the solar wind number density with a power law index of 0.18 , which is calculated from Fig. 4 of Cully et al. (2003a). On the other hand, magnetosheath number density can be approximated to depend linearly on solar wind number density with the typical compression ratio of 4 . Thus, larger contribution of ionosphere is expected to result in lower power law index of the solar wind number density dependence (Fig. 11).

Since ion supply from the ionosphere increases with decreasing distance from the Earth (Cully et al., 2003b), the effect of ionospheric outflow should be most prominent in the near-Earth region. Indeed, our results show that the value of $\eta$ decreases with decreasing distance from the Earth. Ion supply from the ionosphere is also known to depend on the solar wind electric field. This trend is consistent with the fact that faster solar wind under southward IMF result in lower values of $\eta$ (Fig. 12a-b).

Moreover, the value of $\eta$ and $\alpha$ decrease associated with fast solar wind for southward IMF at the high latitude of the midnight region (Fig. 12c-f). These features are likely due to the effect of auroral outflow. The flow velocity in this region is directed tailward and toward the neutral sheet, which means outflow particles are supplied to the near-Earth plasma sheet. Supplied ionospheric particles are expected to be accelerated non-adiabatically (Lyons and Speiser, 1982) and transported into the pre-midnight near-Earth region to result in an increase of plasma number density associated with southward IMF.

If this is the case, the value of $\eta$ is expected to be lower in the pre-midnight near-Earth region than in the post-midnight near-Earth region. However, our result shows that the value of $\eta$ in the near-Earth region is lower in the post-midnight region during fast solar wind and southward IMF conditions. In order to explain this feature, we should consider the effect of gradient-curvature drift on solar wind origin ions transported from the tail. Effect of gradient-curvature drift prevents high-energy ions from moving into the near-Earth postmidnight region, which results in less supply of solar wind particle thus lower values of $\eta$ in the post-midnight region (Fig. 12g).

We should note an unsolved problem concerning the plasma sheet density behavior in the post-midnight region. Wang et al. (2001) modeled mass transport in the near-Earth magnetosphere based on the assumption of slow adiabatic transport. Their result shows that plasma source along the dawn magnetopause is necessary to reproduce the distribution of plasma number density. This dawn flank plasma 


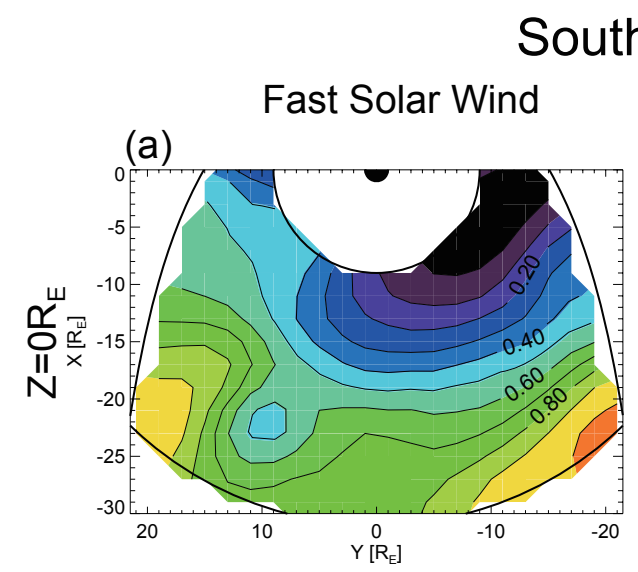

\section{Southward IMF}
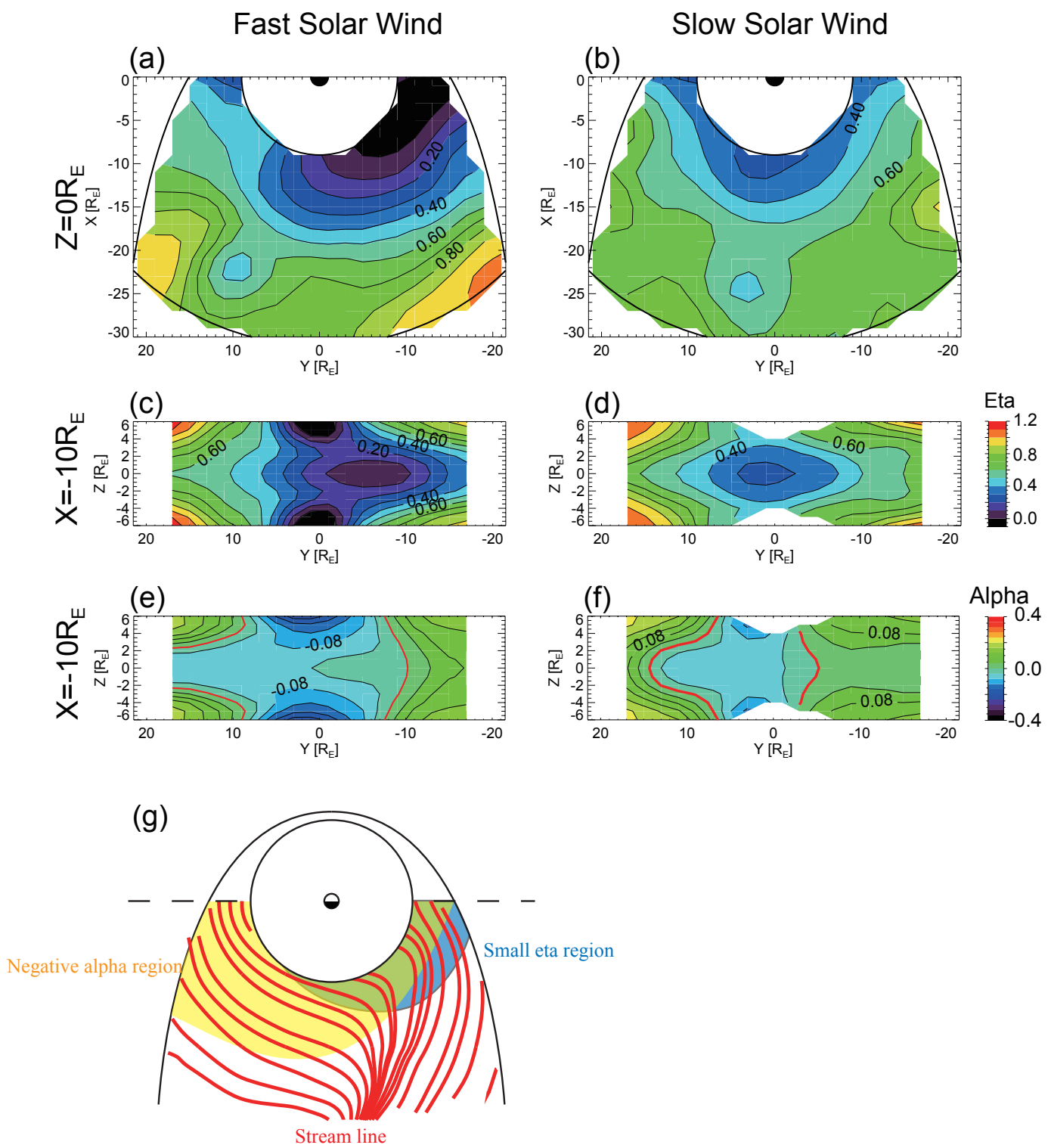

Fig. 12. (a-d) solar wind number density dependence under southward IMF condition for fast and slow solar wind conditions. (e-f) IMF $B_{z}$ dependence at $X=-10 R_{E}$ for fast and slow solar wind conditions. (g) Schematic picture of plasma transport in the near-Earth plasma sheet.

source has been attributed to the magnetosheath. Hasegawa et al. (2003) reported that ion temperature is higher in the perpendicular direction than in the parallel direction to magnetic field, whereas electron temperature shows an opposite anisotropy in the flank region. This fact is consistent with the entry mechanism of magnetosheath particles by kinetic Alfvén wave (Johnson and Cheng, 2003).

At the inner edge of the plasma sheet, Los Alamos National Laboratory instruments in geosynchronous orbit observed cold dense plasma in the dawn region after southward IMF (Lavraud et al., 2006). The perpendicular proton temperature is higher than parallel to the magnetic field there
(Denton et al., 2005). This fact indicates that these particles are not of ionospheric origin. Thus ionospheric contribution to the near-Earth dense plasma is also questionable.

The above observational evidences are inconsistent with our interpretation that the dawn side near-Earth plasma sheet particles are dominantly supplied from the ionosphere. This discrepancy can be attributed to the temporal variation due to substorm activity. Wing et al. (2007) shows plasma sheet properties are dynamically variable in the course of substorm activities. Plasma in the near-Earth dawn region is cold and dense during the growth phase of substorm whereas it is hot and tenuous after the onset of substorm. This large variation 


\section{Southward IMF}

\section{Slow Solar Wind}

(a)

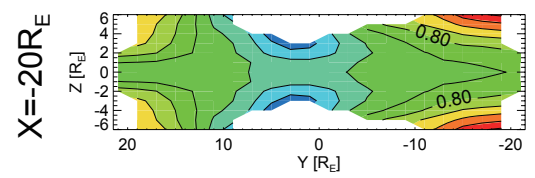

(d)

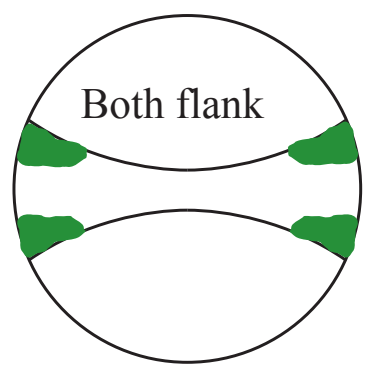

(g)

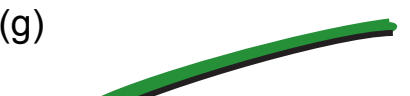

Open high latitude flux tubes

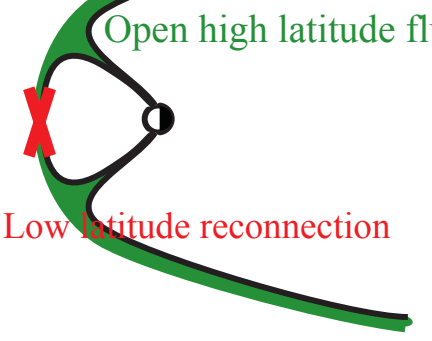

Slow Solar Wind

(b)

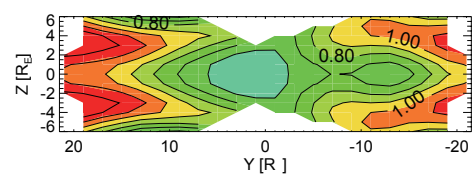

(e)

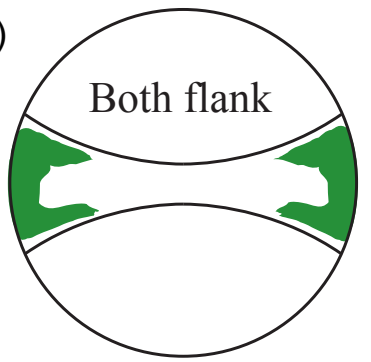

(h)

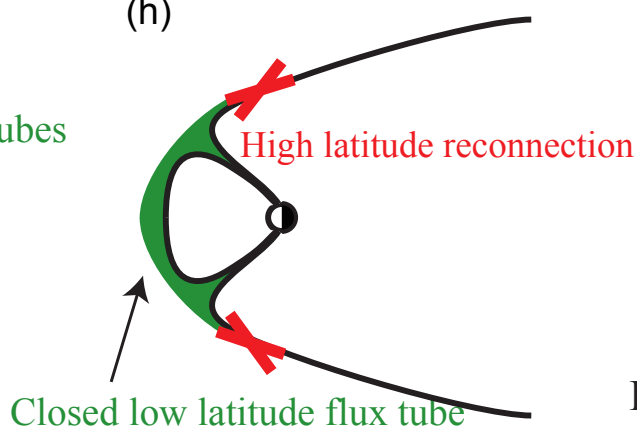

Fast Solar Wind

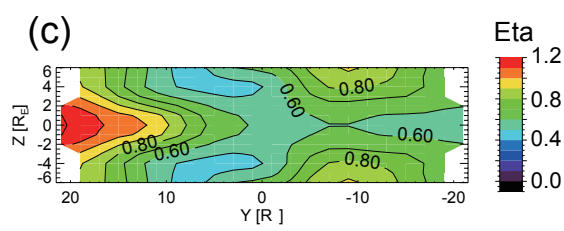

(f)

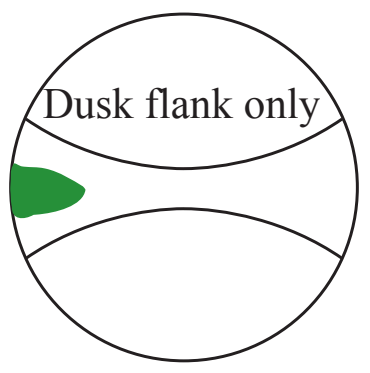

(i)

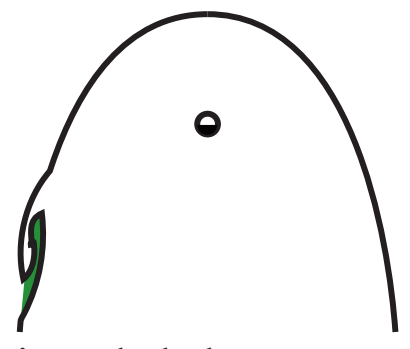

Kelvin-Helmholtz vortex

Fig. 13. Upper raw is cross-section view of plasma sheet from the tail side at $X=-20 R_{E}$. Middle raw is schematic picture for upper raw. Lower raw is locations of $\mathrm{KH}$ vortex and magnetic reconnection. Location of reconnection is represented by a red cross. Captured magnetosheath plasma and flux tubes are colored by green.

of plasma sheet associated with magnetospheric substorm may cause some errors to our analysis.

\subsection{Transport mechanisms of magnetosheath particles into plasma sheet}

Ionospheric particle supply decreases with increasing distance from the Earth (Cully et al., 2003a). Thus, entry mechanisms of magnetosheath particles play dominant roles in determining plasma number density at the distant part of the near-Earth plasma sheet. If the power law index $\eta$ represents the contribution of magnetosheath particles to plasma sheet plasma, the region with large value of $\eta$ is the candidate for entry region.

To our knowledge, there are two theoretical classes of entry mechanisms: one is via magnetic reconnection and the other is via diffusion across the magnetopause. Plasma entry via magnetic reconnection at the magnetopause has different modes for northward and southward IMF conditions. Southward IMF leads to magnetic reconnection at the low-latitude portion of dayside magnetopause and magnetosheath particle entry into the high latitude magnetosphere. Under northward IMF condition, on the other hand, magnetic reconnection occurs at the poleward-of-cusp region and magnetosheath particles are captured in the closed flux tube near the low-latitude magnetopause.

For diffusive entry, various mechanisms such as KelvinHelmhortz $(\mathrm{KH})$ vortex decay, particle scattering by kinetic Alfvén waves (KAWs), and particle entry by gradientcurvature drift (GDE) are proposed (e.g. Nakamura et al., 2004; Johnson and Cheng, 2003; Olson and Pfitzer, 1985, for each mechanism). These mechanisms have different dawndusk asymmetries, which can be used for their identification. Theoretical predictions described above should be tested by the results of observation. 
Under southward IMF conditions, the value of $\eta$ is larger at the high latitude portion of the both dawn and dusk flank regions (Fig. 13a), which is consistent with magnetosheath particle entry from high-latitude magnetosphere by the low latitude dayside reconnection entry mechanism (Fig. 13d, g). A similar structure is seen during the slow solar wind under northward IMF condition. However, the position of the peak is located at $1-2 R_{E}$ closer to the neutral sheet than that under southward IMF condition (Fig. 13b). This feature can be attributed to the formation of closed flux tube with magnetosheath particles formed by high-latitude reconnection under northward IMF (Fig. 13h).

On the other hand, the value of $\eta$ has its peak at the equator of only dusk side flank under fast solar wind conditions (Fig. 13c). This suggests that mechanisms other than reconnection are involved. Since this equatorial peak of $\eta$ is associated with fast solar wind and located in the dusk downtail region, this structure is attributable to the diffusive entry associated with KH instability (Fig. 13i). The growth rate of $\mathrm{KH}$ wave is thought to be larger at low-latitude than highlatitude because of the absence of stabilizing effect of lobe magnetic field. The dawn-dusk asymmetry can be explained by preferential decay of MHD-scale KH vortex due to electron inertia effect (Nakamura et al., 2004).

Comparing the results for Parker and ortho-Parker IMF cases, the value of $\eta$ at the flank region is enhanced at the dawn (dusk) side during (ortho-)Parker IMF orientation (Fig. 14). In other words, the solar wind number density dependence is enhanced in the downstream of quasi-parallel bow shock. Since CLUSTER observations showed distribution of magnetosheath average density is independent from the IMF orientation (V. Génot, private communication), this dawn-dusk asymmetry is likely due to the entry mechanism. The polarity of this switching dawn-dusk asymmetry supports the KAW diffusive entry rather than the GDE entry mechanism. It is also possible that enhanced fluctuation in the magnetosheath affects the frequency of magnetic reconnection at the magnetopause and results in this asymmetry (D. G. Sibeck, private communication).

\subsection{Particle transport in the near-Earth plasma sheet}

For different IMF $B_{z}$ conditions, the plasma sheet has different response time lags to IMF $B_{z}$ variations. Our result also shows that structures of time lag and duration time of IMF $B_{z}$ effects are different for the two IMF $B_{z}$ conditions. In this subsection, we discuss the relation between the IMF $B_{z}$ effect timescale and particle transport in the near-Earth plasma sheet.

Under southward IMF, distribution of time lag for IMF $B_{z}$ effect has clear structure such that the value of time lag increases from the distant region toward both near-Earth flank regions (Fig. 15a). The sign of IMF $B_{z}$ dependence switches at roughly $23 R_{E}$ distance from the Earth (Fig. 6b). In the distant midnight region, southward IMF $B_{z}$ effect appears

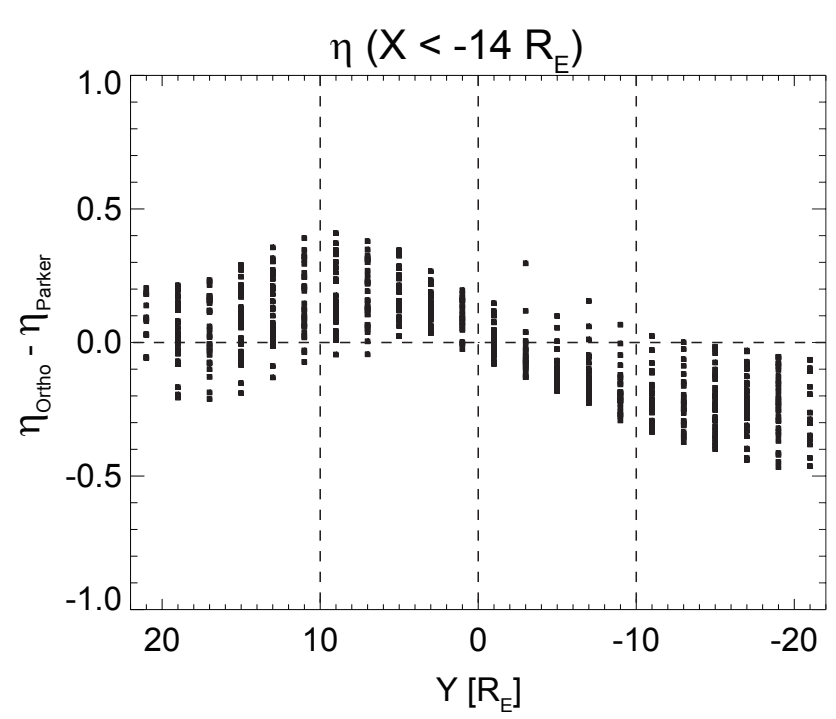

Fig. 14. $Y$ profile of difference of solar wind dependence parameter between Parker and ortho-Parker IMF orientations at $X<-14 R_{E}$.

within $2 \mathrm{~h}$ and the plasma number density decreases. Subsequently, the plasma number density in the nearer portion of the plasma sheet increases with a few hours delay.

This variation of plasma number density suggests reconfiguration of plasma sheet which is caused by plasma transport from distant to near-Earth regions associated with enhanced convection. Figure $15 \mathrm{~b}$ shows distribution of average ion bulk velocity in the equatorial plane without flows faster than $200 \mathrm{~km} / \mathrm{s}$. The value of time lag seems to increase along the stream lines. This fact supports that the effect of IMF $B_{z}$ propagates in forms of convective transport of particles. Average ion temperature shows that plasma is heated during the transport from the distant region to the inner magnetosphere.

Under northward IMF, the distribution of IMF $B_{z}$ effect duration time has interesting structures. If we neglect the peak around $(X, Y)=(-20,-3) R_{E}$, the value increases from distant dawn flank region to near-Earth dusk flank region as a whole (Fig. 16a). The value of duration time again increases along the average ion bulk stream lines (Fig. 16b). This indicates that the effect of IMF $B_{z}$ is flushed away by the plasma convection. In other words, increased plasma number density associated with northward IMF eventually decreases to the nominal value after several hours.

A remaining feature is the peak around $(X, Y)=(-20$, $-3) R_{E}$. Around this peak, fast intermittent flows are frequently observed (Angelopoulos et al., 1994) and flow fluctuations are also large. In such situation, turbulent diffusion might be important to understand the plasma transport in the plasma sheet. In order to interpret the physical meaning of this long duration time area, let us consider the contribution of transport mediated by turbulent diffusion.

In order to estimate turbulent diffusion, we need distributions of plasma number density and diffusion coefficient. We 

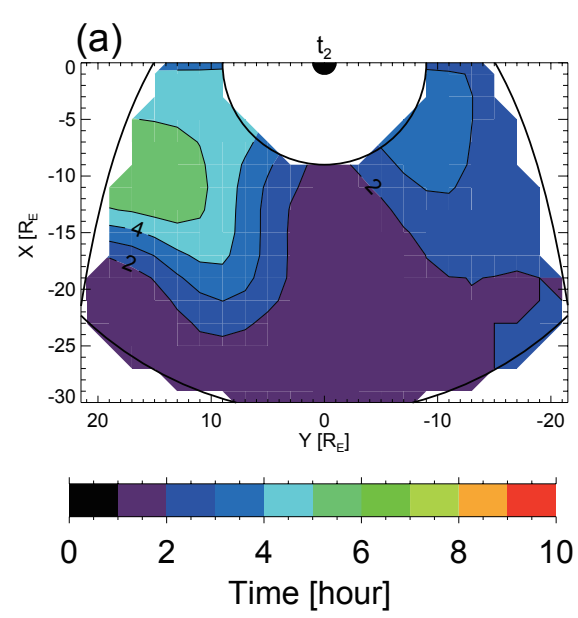
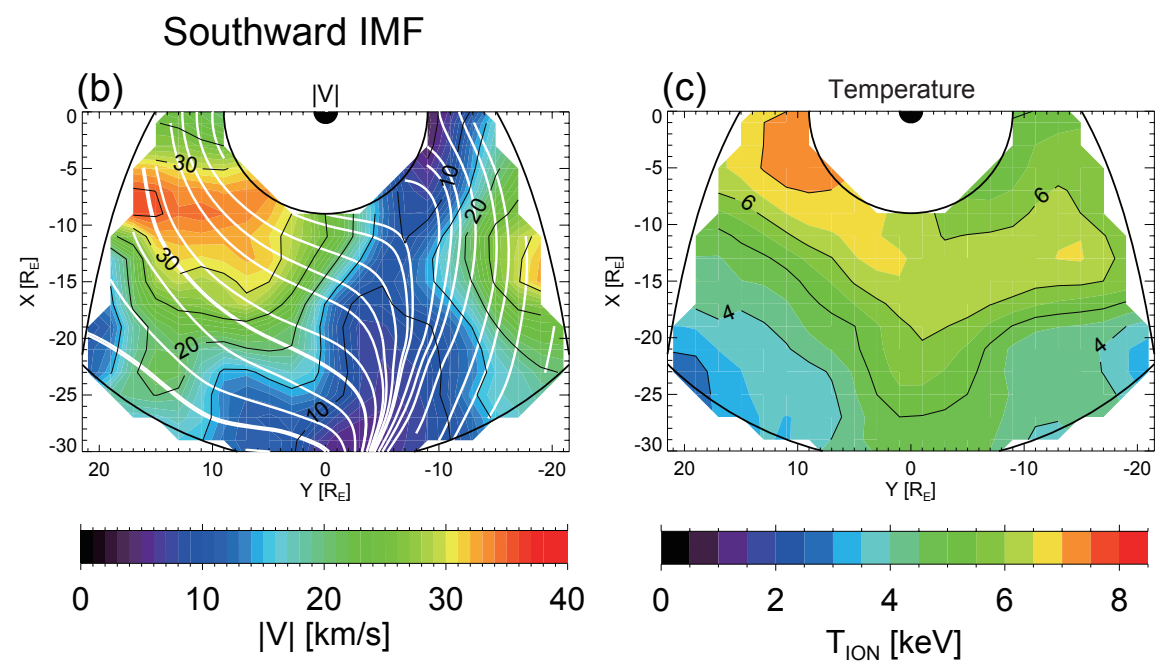

Fig. 15. (a) Equatorial distribution of IMF $B_{z}$ effect time lag for southward IMF. (b) Equatorial distribution of ion flow velocity. Stream lines are shown in white curves. (c) Distribution of average ion temperature.

can use $n_{0}$ for the distribution of plasma number density. We estimated diffusion coefficient by the method of Borovsky et al. (1998) which uses flow fluctuation amplitude and autocorrelation time of flow in the dawn-dusk direction. Diffusion coefficient is estimated by the following equation.

$D=\frac{v_{\mathrm{rms}}^{2} \tau_{\mathrm{corr}}}{2}$

Our result is shown in Fig. 16d. According to Borovsky et al. (1998), turbulent fluctuation is typically $60 \mathrm{~km} / \mathrm{s}$ amplitude and $140 \mathrm{~s}$ duration, which gives the diffusion coefficient of $2.5 \times 10^{5} \mathrm{~km}^{2} / \mathrm{s}$ around $X=-20 R_{E}$. Our result is consistent with theirs despite of the long separation of two observations (i.e. ISEE and GEOTAIL). Diffusion coefficient is large at the distant midnight region for longer autocorrelation time and larger flow fluctuations.

Combined with the distribution of plasma number density, we obtained the diffusion speed as follows.

$V_{\text {diff }}=D \frac{\nabla n_{0}}{n_{0}}$

Due to the large value of number density gradient, diffusion velocity is as large as $2.3 \mathrm{~km} / \mathrm{s}$ in the midnight region at $X=-20 R_{E}$ (Fig. 16e). We also calculated the ratio between diffusion speed and average ion bulk speed (Fig. 16f). Diffusion speed and bulk speed is comparable around $(X$, $Y)=(-25,-7) R_{E}$, which locates next to the peak of IMF $B_{z}$ effect duration time.

Since average bulk flow is directed Earthward while diffusion is estimated to be directed anti-Earthward, these two transports ought to canceled each other. Under normal situation, convective bulk flow overcomes the diffusive transport. However, the diffusive transport by turbulence can affect the convective transport during weak convection such as under northward IMF condition. We propose that this cancellation results in the peak of IMF $B_{z}$ effect duration. Contribution from numerical modeling with realistic turbulence effects to examine this phenomenon is awaited in the future.

\subsection{Summary}

We examined three-dimensional structures of dependences of plasma sheet number density on solar wind number density and IMF $B_{z}$ by fitting the observations to an empirical model. Effects of IMF $B_{z}$ polarity in 10-h time scale, solar wind speed, and IMF orientation were also investigated by subdivision of dataset. Based on obtained features of dependence parameters' distributions, we discussed the contribution of outflow from the ionosphere and entry mechanisms of magnetosheath particles.

In the near-Earth region, dependence on solar wind number density is weak for the southward IMF and faster solar wind condition. We interpret this feature due to contribution of mass supply from the terrestrial ionosphere which weakly depends on solar wind number density. Weak dependence on solar wind number density and strong dependence on IMF $B_{z}$ in the midnight high-latitude region are attributable to the auroral outflow. Dawn-dusk asymmetry of solar wind number density dependence in the near-Earth region can be associated with substorm dynamics yet remains to be open.

Further from the Earth, dependence on solar wind number density is different for different solar wind conditions. The dependence is strong at the flank high-latitude region for southward IMF condition. Entry of magnetosheath particles associated with low-latitude magnetopause reconnection is a plausible candidate for this feature. Under northward IMF and slow solar wind condition, the region of dependence is located at lower latitude. This suggests the relevance of 

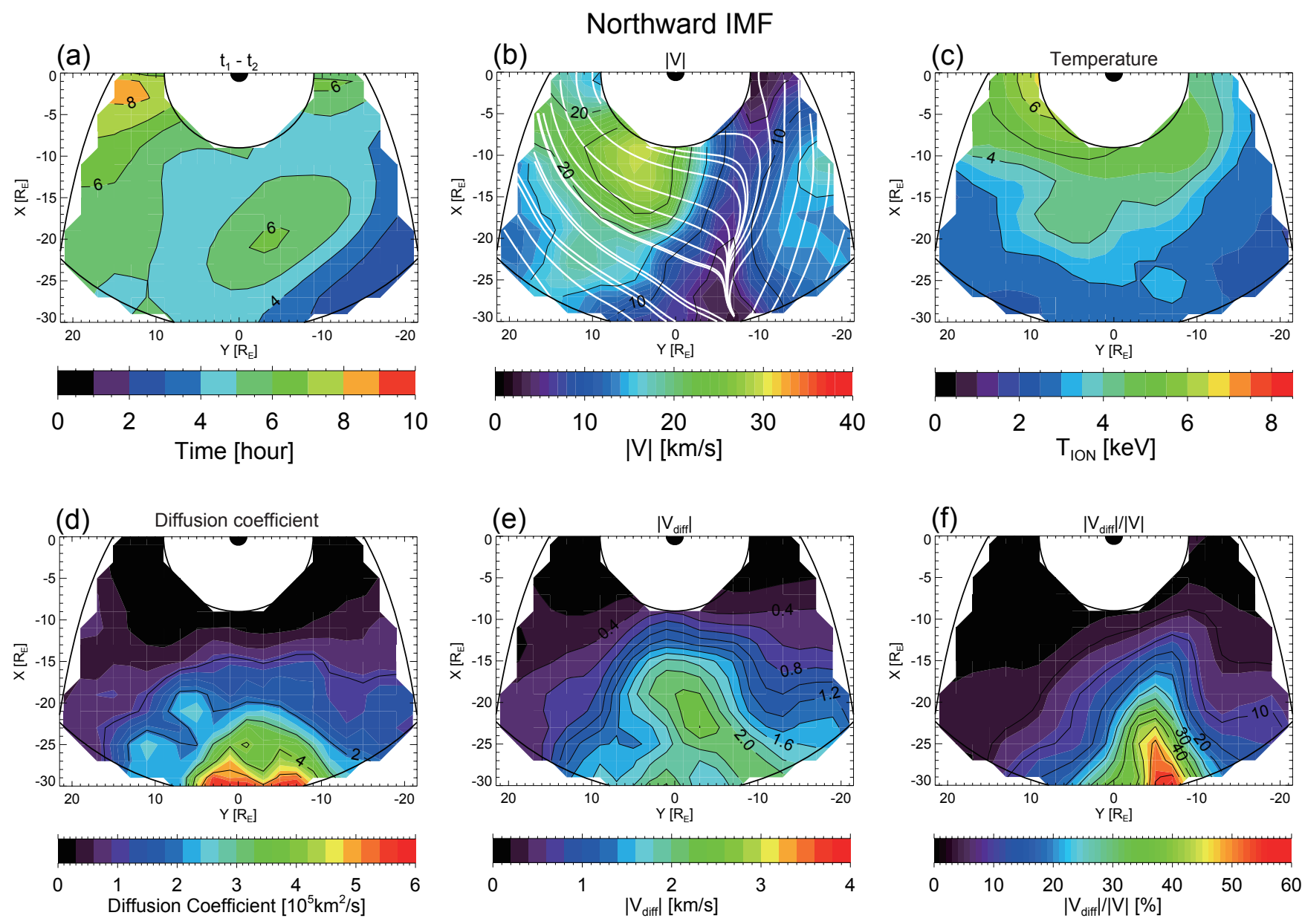

Fig. 16. (a) Equatorial distribution of IMF $B_{z}$ effect duration time for northward IMF. (b) Equatorial distribution of ion flow velocity. Stream lines are shown in white curves. (c) Distribution of average ion temperature. (d) Distribution of diffusion coefficient. (e) Distribution of diffusion speed. (f) Distribution of ration between diffusion speed and average ion bulk speed.

high-latitude reconnection and the resultant capture of magnetosheath plasma.

For faster solar wind condition under northward IMF, the strong dependence region is limited in the dusk flank neutral sheet. Magnetosheath particle entry via Kelvin-Helmholtz vortex decay can explain this feature. IMF orientation angle seems to influence the dawn-dusk asymmetry of the solar wind number density dependence in the distant region. The polarity of asymmetry suggests the influence of diffusive entry via kinetic Alfven wave at the magnetopause.

We also discussed the distribution of IMF $B_{z}$ effect time lags. Under southward IMF, distribution of IMF $B_{z}$ effect time lag can be understood by the convective transport which causes reconfiguration of the near-Earth plasma sheet. Under northward IMF condition, distribution of IMF $B_{z}$ duration time has a similar structure with that of time lag under southward IMF condition. This feature is also attributable to the effect of plasma convection. In addition to this structure, there is a peak of IMF $B_{z}$ effect duration time in the middle of examined area. We proposed that this structure is related with diffusive transport by turbulence in the plasma sheet.

Acknowledgements. EPIC-STICS data was provided by Steve P. Christon, Richard W. McEntire, and Masahito Nosé. MGF data was provided by Tsugunobu Nagai of Tokyo Institute of Technology. The high resolution OMNI solar wind data was provided by Joe King and Natalia Papitashvili of NASA/GSFC. This work is supported by the JSPS grant for young scientists.

Topical Editor I. A. Daglis thanks two anonymous referees for their help in evaluating this paper.

\section{References}

Angelopoulos, V., Kennel, C. F., Coroniti, F. V., Pellat, R., Kivelson, M. G., Walker, R. J., Russell, C. T., Baumjohann, W., Feldman, W. C., and Gosling, J. T.: Statistical Characteristics of Bursty Bulk Flow Events, J. Geophys. Res., 99(A11), 21 257$21280,1994$. 
Borovsky, J. E., Thomsen, M. F., and Elphic, R. C.: The driving of the plasma sheet by the solar wind, J. Geophys. Res., 103(A8), 17 617-17 640, 1998.

Chappell, C. R., Moore, T. E., and Waite Jr., J. H.: The ionosphere as a fully adequate source of plasma for the Earth's magnetosphere, J. Geophys. Res., 92(A6), 5896-5910, 1987.

Cully, C. M., Donovan, E. F., Yau, A. W., and Opgenoorth, H. J.: Supply of thermal ionospheric ions to the central plasma sheet, J. Geophys. Res., 108(A2), 1092, doi:10.1029/2002JA009457, 2003a.

Cully, C. M., Donovan, E. F., Yau, A. W., and Arkos, G. G.: Akebono/Suprathermal Mass Spectrometer observations of low-energy ion outflow: Dependence on magnetic activity and solar wind conditions, J. Geophys. Res., 108(A2), 1093, doi:10.1029/2001JA009200, 2003b.

Denton, M. H., Thomsen, M. F., Korth, H., Lynch, S., Zhang, J. C., and Liemohn, M. W.: Bulk plasma properties at geosynchronous orbit, J. Geophys. Res., 110, A07223, doi:10.1029/2004JA010861, 2005.

Frey, H. U., Mende, S. B., Angelopoulos, V., and Donovan, E. F.: Substorm onset observations by IMAGE-FUV, J. Geophys. Res., 109, A10304, doi:10.1029/2004JA010607, 2004.

Hasegawa, H., Fujimoto, M., Maezawa, K., Saito, Y., and Mukai, T.: Geotail observations of the dayside outer boundary region: Interplanetary magnetic field control and dawn-dusk asymmetry, J. Geophys. Res., 108(A4), 1163, doi:10.1029/2002JA009667, 2003.

Johnson, J. R. and Cheng, C. Z.: Kinetic Alfvén waves at the magnetopause - mode conversion, transport and formation of LLBL, in Earth's low-latitude boundary layer, Geophysical Monograph 133, American Geophysical Union, Washington, D.C., 2003.

Kaufmann, R. L., Lu, C., Paterson, W. R., and Frank, L. A.: Three-dimensional analyses of electric currents and pressure anisotropies in the plasma sheet, J. Geophys. Res., 107(A7), 1103, doi:10.1029/2001JA000288, 2002.

Kaufmann, R. L., Paterson, W. R., and Frank, L. A.: Birkeland currents in the plasma sheet, J. Geophys. Res., 108(A7), 1299, doi:10.1029/2002JA009665, 2003.

Kaufmann, R. L., Paterson, W. R., and Frank, L. A.: Pressure, volume, density relationships in the plasma sheet, J. Geophys. Res., 109, A08204, doi:10.1029/2003JA010317, 2004.

Kaufmann, R. L., Paterson, W. R., and Frank, L. A.: Magnetization of the plasma sheet, J. Geophys. Res., 109, A09212, doi:10.1029/2003JA010148, 2004.

Kaufmann, R. L., Paterson, W. R., and Frank, L. A.: Relationships between the ion flow speed, magnetic flux transport rate, and other plasma sheet parameters, J. Geophys. Res., 110, A09216, doi:10.1029/2005JA011068, 2005.

Kaufmann, R. L. and Paterson, W. R.: Magnetic flux and particle transport in the plasma sheet, J. Geophys. Res., 111, A10214, doi:10.1029/2006JA011734, 2006.

Kokubun, S., Yamamoto, T., Acuna, M. H., Hayashi, K., Shiokawa, K., and Kawano, H.: The GEOTAIL magnetic field experiment, J. Geomag. Geoelectr., 46, 7-21, 1994.

Lavraud, B., Thomsen, M. F., Wing, S., Fujimoto, M., Denton, M. H., Borovsky, J. E., Aasnes, A., Seki, K., and Weygand, J. M.: Observation of two distinct cold, dense ion populations at geosynchronous orbit: local time asymmetry, solar wind dependence and origin, Ann. Geophys., 24, 3451-3465, 2006, http://www.ann-geophys.net/24/3451/2006/.

Lennartsson, O. W.: ISEE ion composition data with implications for solar wind entry into Earth's magnetotail, Space Sci. Rev., 80, 305-323, 1992.

Lyons, L. R. and Speiser, T. W.: Evidence for current sheet acceleration in the geomagnetic tail, J. Geophys. Res., 87(A4), 22762286, 1982.

Moore, T. E., Fok, M.-C., Chandler, M., Chappell, C. R., Christon, S. P., Delcourt, D. C., Fedder, J., Huddleston, M., Liemohn, M., Peterson, W. K., and Slinker, S.: Plasma sheet and (nonstorm) ring current formation from solar and polar wind sources, J. Geophys. Res., 110, A02210, doi:10.1029/2004JA010563, 2005.

Mukai, T., Machida, S., Saito, Y., Hirahara, M., Terasawa, T., Kaya, N., Obara, T., Ejiri, M., and Nishida, A.: The low energy particle (LEP) experiment onboard the GEOTAIL satellite, J. Geomag. Geoelectr., 46, 669-692, 1992.

Nagata, D., Machida, S., Ohtani, S., Saito, Y., and Mukai, T.: Solar wind control of plasma number density in the near-Earth plasma sheet, J. Geophys. Res., 112, A09204, doi:10.1029/2007JA012284, 2007.

Nakamura, T. K. M., Hayashi, D., Fujimoto, M., and Shinohara, I.: Decay of MHD-scale Kelvin-Helmholtz vortex mediated by parasitic electron dynamics, Phys. Rev. Lett., 92, 145001, doi:10.1103/PhysRevLett.92.145001, 2004.

Olson, W. P. and Pfitzer, K. A.: Magnetospheric responses to the gradient drift entry of solar wind plasma, J. Geophys. Res., 9, 10 823-10 833, 1985.

Sergeev, V., Runov, A., Baumjohann, W., Nakamura, R., Zhang, T. L., Balogh, A., Louarnd, P., Sauvaud, J.-A., and Reme, H.: Orientation and propagation of current sheet oscillations, Geophys. Res. Lett., 31, L05807, doi:10.1029/2003GL019346, 2004.

Sergeev, V. A., Sormakov, D. A., Apatenkov, S. V., Baumjohann, W., Nakamura, R., Runov, A. V., Mukai, T., and Nagai, T.: Survey of large-amplitude flapping motions in the midtail current sheet, Ann. Geophys., 24, 2015-2024, 2006, http://www.ann-geophys.net/24/2015/2006/.

Shue, J.-H., Song, P., Russell, C., Steinberg, J., Chao, J., Zastenker, G., Vaisberg, O., Kokubun, S., Singer, H., Detman, T., and Kawano, H.: Magnetopause location under extreme solar wind conditions, J. Geophys. Res., 103(A8), 17 691-17 700, 1998.

Terasawa, T., Fujimoto, M., Mukai, T., Shinohara, I., Saito, Y., Yamamoto, T., Machida, S., Kokubun, S., Lazarus, A., Steinberg, J., and Lepping, R.: Solar wind control of density and temperature in the near-Earth plasma sheet: WIND/GEOTAIL collaboration, Geophys. Res. Lett., 24(8), 935-938, 1997.

Tsyganenko, N. A. and Mukai, T.: Tail plasma sheet models derived from Geotail particle data, J. Geophys. Res., 108(A3), 1136, doi:10.1029/2002JA009707, 2003.

Tsyganenko, N. A. and Fairfield, D. H.: Global shape of the magnetotail current sheet as derived from Geotail and Polar data, J. Geophys. Res., 109, A03218, doi:10.1029/2003JA010062, 2004.

Tsyganenko, N. A. and Sitnov, M. I.: Magnetospheric configurations from a high-resolution data-based magnetic field model, J. Geophys. Res., 112, A06225, doi:10.1029/2007JA012260, 2007.

Volwerk, M., Glassmeier, K.-H., Runov, A., Nakamura, R., Baumjohann, W., Klecker, B. Richter, I., Balogh, A., Rème, H., and Yumoto, K.: Flow burst-induced large-scale plasma sheet oscillation, J. Geophys. Res., 109, A11208, doi:10.1029/2004JA010533, 2004. 
Walker, R. J., Ashour-Abdalla, M., Ogino, T., Peroomian, V., and Richard, R. L.: Modeling magnetospheric sources, in: Earth's low-latitude boundary layer, Geophysical Monograph 133, American Geophysical Union, Washington, D.C., 2003.

Wang, C., Lyons, L. R., Chen, M. W., and Wolf, R. A.: Modeling the quiet time inner plasma sheet protons, J. Geophys. Res., 106(A4), 6161-6178, 2001.

Wang, C.-P., Lyons, L. R., Weygand, J. M., Nagai, T., and McEntire, R. W.: Equatorial distributions of the plasma sheet ions, their electric and magnetic drifts, and magnetic fields under different interplanetary magnetic field $B_{z}$ conditions, J. Geophys. Res., 111, A04215, doi:10.1029/2005JA011545, 2006.

Wang, C.-P., Lyons, L. R., Nagai, T., Weygand, J. M., and McEntire, R. W.: Sources, transport, and distributions of plasma sheet ions and electrons and dependences on interplanetary parameters under northward interplanetary magnetic field, J. Geophys. Res., 112, A10224, doi:10.1029/2007JA012522, 2007.
Williams, D. J., McEntire, R. W., Schlemm II, C., et al.: Geotail energetic particles and ion composition instrument, J. Geomagn. Geoelectr., 46, 39-57, 1994.

Wing, S. and Newell, P. T.: 2D plasma sheet ion density and temperature profiles for northward and southward IMF, Geophys. Res. Lett., 29(9), 1307, doi:10.1029/2001GL013950, 2002.

Wing, S., Johnson, J. R., Newell, P. T., and Meng, C.-I.: Dawndusk asymmetries, ion spectra, and sources in the northward interplanetary magnetic field plasma sheet, J. Geophys. Res., 110, A08205, doi:10.1029/2005JA011086, 2005.

Wing, S., Gjerloev, J. W., Johnson, J. R., and Hoffman, R. A.: Substorm plasma sheet ion pressure profiles, Geophys. Res. Lett., 34, L16110, doi:10.1029/2007GL030453, 2007. 